\title{
In-vitro derived germinal centre B cells differentially generate memory B or plasma cells in vivo
}

\author{
Takuya Nojima', Kei Haniuda', Tatsuya Moutai', Moeko Matsudaira', Sho Mizokawa', Ikuo Shiratori', \\ Takachika Azuma² \& Daisuke Kitamura'
}

In response to $T$ cell-dependent antigens, $B$ cells proliferate extensively to form germinal centres $(G C)$, and then differentiate into memory $B\left(B_{\text {mem }}\right)$ cells or long-lived plasma cells (LLPCs) by largely unknown mechanisms. Here we show a new culture system in which mouse naïve $B$ cells undergo massive expansion and isotype switching, and generate GC-phenotype $B(i G B)$ cells. The $i G B$ cells expressing $\lg G 1$ or $\operatorname{lgM} / D$, but not $\lg E$, differentiate into $B_{\text {mem }}$ cells in vivo after adoptive transfer and can elicit rapid immune responses with the help of cognate T cells. Secondary culture with IL-21 maintains the proliferation of the iGB cells, while shifting their in vivo developmental fate from $B_{\text {mem }}$ cells to LLPCs, an outcome that can be reversed by withdrawal of IL-21 in tertiary cultures. Thus, this system enables in vitro manipulation of B-cell fate, into either $B_{\text {mem }}$ cells or LLPCs, and will facilitate dissection of GC-B cell differentiation programs. 
M emory B $\left(\mathrm{B}_{\mathrm{mem}}\right)$ cells and long-lived plasma cells (LLPCs) are the effectors of immunological memory, a hallmark of adoptive immunity, and are crucial to protect the host against recurrent infection. These cells are typically generated in germinal centres (GCs) formed in the peripheral lymphoid tissues during the T-cell-dependent (TD) immune response. In the TD response, antigen (Ag)-specific B cells present the $\mathrm{Ag}$ to primed cognate Th cells in the lymphoid follicles and clonally proliferate on receiving signals such as CD40-ligand (CD40L) and IL-4 from the Th cells. Some of the responding B cells leave the follicles and form extrafollicular foci of plasmablasts, which differentiate into shortlived plasma cells that survive for only a few days. Other B cells proliferate extensively in the follicles with the help of follicular helper T (Tfh) cells to form GCs, in which B cells undergo isotype switching, typically to IgG subclasses, and somatic hypermutation (SHM) of Ig genes to diversify the affinity of their B-cell receptor (BCR) for $\mathrm{Ag}^{1}$. Finally, GC B cells expressing a high-affinity IgG BCR are selected and differentiate into $B_{\text {mem }}$ cells and LLPCs by largely unknown mechanisms ${ }^{2-4}$. Some of $\mathrm{IgG}^{+} \mathrm{GC} B$ cells further switch their BCR to IgE, but the $\operatorname{IgE}^{+}$GC B cells are localized outside GCs and rapidly differentiate into plasma cells $s^{5}$.

In the TD response, the $\mathrm{CD} 40-\mathrm{CD} 40$ ligand $(\mathrm{CD} 40 \mathrm{~L})$ interaction is required for Th-mediated B-cell activation and thus for GC formation, and for generation of $B_{\text {mem }}$ cells $s^{6-8}$. In addition, IL-21 signalling is required for IgG1 production, efficient GC formation, affinity maturation and LLPC formation, but not for $\mathrm{B}_{\mathrm{mem}}$ cell development in mice ${ }^{9-12}$. IL-21 induces class switch recombination (CSR) to $\operatorname{IgG}^{13}$ and Blimp1 expression in B cells through transcriptional activation of the Blimp1-enoding Prdm 1 gene via cooperation of Irf4 and Stat3 (refs 14,15), and thus PC differentiation ${ }^{14,16-18}$. IL-21 was also reported to be required for generation of Th cells, which themselves produce IL-21 (refs 19,20), but this idea has recently been questioned ${ }^{9-11}$. Numerous studies have shown that the transcription factor Bcl-6 is essential for the differentiation of Tfh cells ${ }^{21}$ and GC B cells, affinity maturation and LLPC formation, but not for $\mathrm{B}_{\text {mem }}$ cell development ${ }^{22}$. Together, these data suggest a model in which GC B-cell differentiation, which is dependent on IL-21 signalling and $\mathrm{Bcl}-6$, is a necessary prerequisite for production of the high-affinity $B_{\text {mem }}$ cells and LLPCs, but is not essential for $B_{\text {mem }}$ cell generation per se. Thus, signalling and transcriptional programs that determine $B_{\text {mem }}$ cell fate are still unknown. Similarly, it remains unclear what determines lineage choice by the affinity-selected GC B cells to become LLPCs, although transcriptional regulators such as Blimp-1 and Irf4 are known to be essential for overall plasma cell differentiation ${ }^{23,24}$.

A study to elucidate molecular mechanisms for the fate decision of GC B cells in mice is challenging, because the numbers of $\mathrm{B}_{\mathrm{mem}}$ cells and LLPCs, and presumably their precursors in GCs, are too few to be experimentally manipulated ex vivo. Moreover, there is no definitive marker for $\mathrm{B}_{\text {mem }}$ cells in mice. B7.1 (CD80) ${ }^{25}$ and CD38 (ref. 26) are useful but not $B_{\text {mem }}$-specific markers. Thus, $B_{\text {mem }}$ cells have been identified as B cells expressing Ag-specific IgG in immunized mice, but $B_{\text {mem }}$ cells bearing unswitched BCR $\left(\operatorname{IgM}^{+}\right.$ $\mathrm{IgD}^{+}$) have also been recognized ${ }^{27-29}$. Functionally, $\mathrm{B}_{\text {mem }}$ cells can be defined as long-lived Ag-experienced B cells that rapidly produce $\mathrm{Ab}$ in response to re-challenge with soluble, adjuvant-free Ags after being transferred into recipient mice together with cognate $\mathrm{Th}$ $\operatorname{cell}^{30}$. This assay should be potentially useful to identify factors that affect development, longevity, in situ localization, Ag responsiveness and so on of $B_{m e m}$ cells, but their paucity and in vitro fragility have limited these applications, except for using specific mutant mouse strains $^{27,31,32}$.

In vitro culture systems that allow long-term proliferation of primary $\mathrm{T}$ cells and their differentiation towards specific $\mathrm{T}$ cell subsets, for example, Th1, Th2, Th17 or Tregs, have been established and broadly used to study molecular mechanisms for T-cell differentiation. A culture system for human B cells, in which tonsil GC B cells can differentiate to either the PC or $B_{\text {mem }}$ phenotype, was previously reported ${ }^{33-35}$, although functional studies of the human $B_{\text {mem }}$ cells remain extremely difficult. Mouse B cells are known to proliferate, undergo class switching and differentiate into plasma cells in response to stimulation with anti-Ig Ab, LPS, anti-CD40 Ab, and/or cytokines, such as IL-4, in vitro, but the resulting cell growth is transient and the cells die within days. Thus, there has been no practical in vitro system to propagate B cells and then control their differentiation towards $B_{\text {mem }}$ cell or LLPC lineages.

Here we describe a new in vitro culture system in which naïve murine $B$ cells proliferate enormously, exhibit a GC B cell phenotype, and develop into either $\mathrm{B}_{\mathrm{mem}}$ cells or LLPCs in vivo after adoptive transfer. With this system, we have clearly demonstrated a role for IL-21 in determination of the GC B cell fate, the reversible suppression of $\mathrm{B}_{\mathrm{mem}}$ cell commitment.

\section{Results}

A new culture system to propagate GC phenotype B cells. To propagate B cells in vitro, we generated three feeder cell lines: BALB/c 3T3 fibroblasts stably transfected with both CD40L and BAFF (40LB) (Fig. 1a) or individually with either CD40L (3T3/40L) or BAFF (3T3/BAFF). We cultured naïve splenic B cells on these feeder cells in the presence of IL-4. The B cells proliferated on 40LB cells and the live cell number increased, resulting in a 180fold expansion after 6 days (Fig. 1b), whereas there was minimal proliferation on $3 \mathrm{~T} 3 / 40 \mathrm{~L}, 3 \mathrm{~T} 3 / \mathrm{BAFF}$ or $3 \mathrm{~T} 3$ cells. On $3 \mathrm{~T} 3 / \mathrm{BAFF}$ cells, the B cell number increased less than tenfold by co-stimulation with anti-CD40 Ab and IL-4, and the expansion was even less with IL-4 and LPS (Fig. 1b). These differences in expansion were due partly to differences in DNA synthesis as demonstrated by BrdU incorporation (Fig. 1c). CFSE-labelling analysis also revealed that the all B cells replicated more rapidly on 40LB cells with IL-4 than without, whereas B cells on 3T3/BAFF with anti-CD40 and IL-4 replicated more slowly and heterogeneously (Fig. 1d). These data indicate that co-expression of membrane-bound CD40L and BAFF is required for the feeder cells to support optimum proliferation of B cells.

We next analysed the phenotype of the B cells growing on 40LB by flow cytometry. As expected, IL-4 induced BCR class switching from IgM to IgG1 and IgE, generating discrete populations of IgG1 ${ }^{+}$ and $\mathrm{IgE}^{+}$cells by day 4 (Fig. 1e). The $\mathrm{IgE}^{+}$cell population further increased in frequency with time, whereas the $\mathrm{IgG1}^{-} \mathrm{IgE}^{-}$cell population, consisting mostly of $\mathrm{IgM}^{+} \mathrm{IgD}^{+}$cells, decreased. A small fraction of the IgG1 ${ }^{-}$cells (mostly $\operatorname{IgE}^{+}$) were $\mathrm{CD} 138^{+}$and these increased in frequency with time. All but the $\mathrm{CD} 138^{+}$cells exhibited a cell-surface phenotype typical of GC B cells: $\mathrm{GL}^{+} \mathrm{Fas}^{+} \mathrm{CD} 38^{\text {low }}$ and $\mathrm{PNA}^{+}$(Fig. 1e), comparable to ex vivo GC B cells in the spleen of immunized mice (Fig. 1f). The expression of CCR7 on the iGB cells was as similar to naïve follicular B cells (Supplementary Fig. $\mathrm{S} 1$ ), as reported for mouse $\mathrm{GC} \mathrm{B}^{36}$, and unlike the high CCR7 levels seen on activated $B$ cells ${ }^{37}$. Thus, we call these $B$ cells propagated on 40LB cells in-vitro-induced GC B (iGB) cells.

The $\mathrm{IgG1}^{+}, \mathrm{IgE}^{+}$or $\mathrm{IgM}^{+}, \mathrm{CD} 138^{-}$populations of $\mathrm{iGB}$ cells on day 4 expressed abundant Bcl- 6 and $\mathrm{Bcl}-\mathrm{xL}$ proteins, at a level even higher than the ex vivo GC B cells (Fig. 1g). Both proteins have recently been shown to be significantly higher in mouse GC $B$ cells than in follicular B cells ${ }^{38}$, showing that iGB cells share some molecular features with GC B cells.

Generation of $B_{\text {mem }}$ cells from iGB cells in vivo. $B_{\text {mem }}$ cells typically develop from GC B cells, but $B_{\text {mem }}$ cells with unmutated Igs can develop in mice lacking discernable $\mathrm{GCs}^{27}$. Therefore, we assessed whether the iGB cells contain $B_{\text {mem }}$ cell precursors. iGB cells expressing an allelic marker (Ly5.1 $1^{+}$) were transferred intravenous (i.v.) 

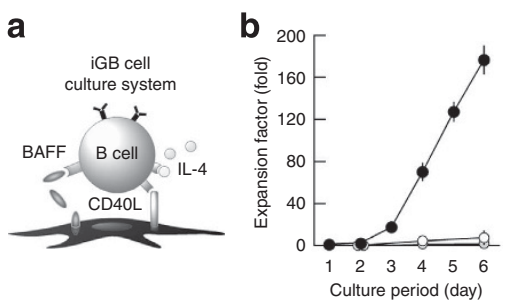

C

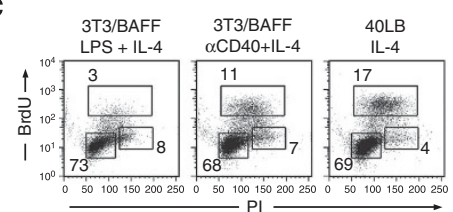

d

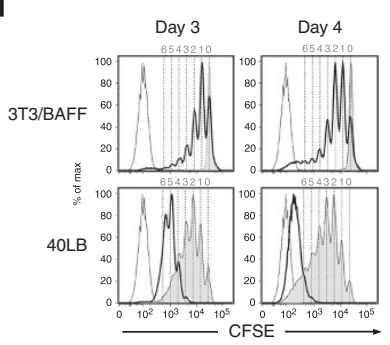

f

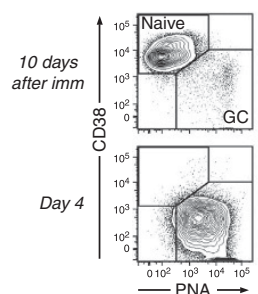

e
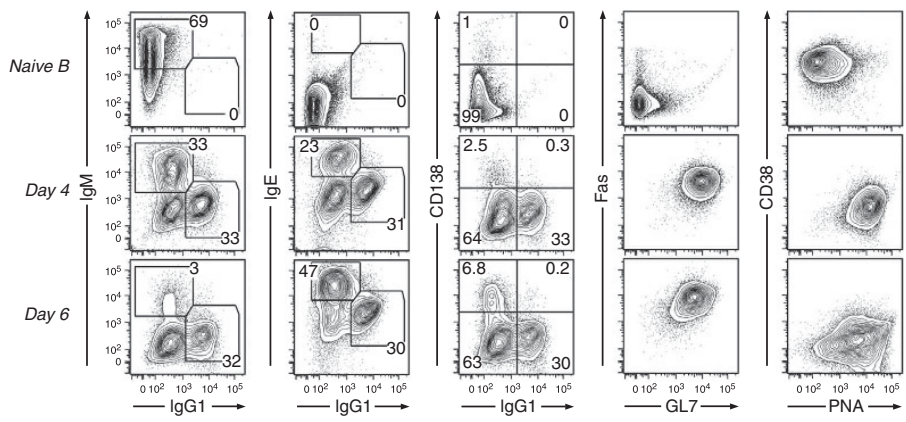

Figure 1 | In vitro induction and massive expansion of phenotypically GC B cells from naïve B cells. (a) Schematic representation of the iGB cell culture system. (b) Cumulative fold increase in the number of live B cells cultured on 4OLB with IL-4 (closed circles), on 3T3/BAFF with anti-CD40 Ab (1 $\mu$ g ml ${ }^{-1}$ ) plus IL-4 (open circles), or on 3T3/BAFF with LPS $\left(10 \mu \mathrm{g} \mathrm{ml}^{-1}\right)$ plus IL-4 (shaded circles). Shown are mean values with s.d. at the indicated time points from three independent experiments, each done singly from an individual mouse. (c) B cells were cultured as in (b) for 3 days and pulsed with BrdU for the last $1 \mathrm{~h}$. The cells were stained with anti-BrdU Ab and propidium iodide and analysed by flow cytometry. Numbers indicate the percentages of the cells in the G1 (lower left window), S (upper window), and G2-M (lower right window) phases of cell cycle. The data shown are representative of two independent experiments. (d) CFSE-labelled splenic naïve B cells were cultured for 3 or 4 days on 3T3/BAFF with anti-CD40 Ab (1 $\mu$ g ml $\left.{ }^{-1}\right)$ plus IL-4 (thick line) or without (shaded) ('3T3/BAFF'), or on $40 \mathrm{LB}$ with IL-4 (thick line) or without (shaded) ('4OLB'), and then analysed by flow cytometry. Unlabelled B cells cultured on $40 \mathrm{LB}$ with IL-4 served as negative controls (thin line). Theoretical cell division numbers are noted on top of each histogram. The data shown are representative of three independent experiments. (e) Flow cytometric analysis for the indicated markers of iGB cells cultured with IL-4 for 4 or 6 days, or of splenic naïve B cells from a C57BL/6 mouse. Expression of Fas, GL7, CD38, and PNA ligand was analysed for CD138- gated cells. Numbers indicate the percentages of cells in the indicated quadrants or windows. The data shown are representative of numerous independent experiments. (f) Flow cytometric analysis of iGB cells cultured with IL-4 for 4 days (day 4), and of pooled splenic B cells from three mice that had been immunized with $100 \mu \mathrm{g}$ of NP-CGG/alum 10 days before (10 days after imm). Expression of CD38, PNA ligand, Fas and GL7 on CD19+ CD138- gated cells is shown. (g) Western blot analysis was performed using the following cells purified by the FACSAria II cell sorter: $\operatorname{lgG} 1^{+}, \lg \mathrm{IM}^{+}$or $\operatorname{lgE}{ }^{+}$, CD138- populations from iGB cells 4 days after primary culture with IL-4 (iGB-4), and $\mathrm{IgG1} 1^{+}$or $\mathrm{IgE} \mathrm{E}^{+}, \mathrm{CD} 138^{-}$populations from those after the subsequent culture for 2 more days with IL-21 (iGB-21), splenic GC B cells (GC: GL7+ ${ }^{+} \mathrm{Fas}^{+}, \mathrm{CD}^{+}{ }^{+}, \mathrm{CD}_{138}{ }^{-}$) from 20 C57BL/6 mice immunized with BSA/CFA 2 weeks earlier, splenic naïve follicular B cells (NF: $\mathrm{CD}_{21}{ }^{+}, \mathrm{CD}_{2} 3^{\text {high }}, \mathrm{CD}_{19}{ }^{+}, \mathrm{CD}^{-} 3^{-}$) from unimmunized $\mathrm{C} 57 \mathrm{BL} / 6$ mice. IgG ${ }^{+}$mouse B-lymphoma cell line A20 was used as a control. All samples consist of $2 \times 10^{6}$ cells per lane, except the $\lg \mathrm{M}^{+} \mathrm{iGB}-4$ ells $\left(1 \times 10^{6} \mathrm{cells}\right)$. The blot was probed with antibodies against indicated proteins.

into irradiated C57BL/6 (Ly5.2+ $2^{+}$mice. Eight weeks later, a significant population of donor-derived B cells $\left(\right.$ Ly5 $\left.5.1^{+}, \mathrm{CD} 19^{+}\right)$was detected in the spleen (Fig. 2a). In contrast to the recipient $\mathrm{B}$ cells, which were uniformly IgM/ $\mathrm{D}^{+}$, about two-thirds of these cells were $\operatorname{IgG} 1^{+}$, and the rest were $\operatorname{IgM}^{+} \operatorname{IgD}^{+}\left(\operatorname{IgM} / \mathrm{D}^{+}\right)$. Notably, donorderived $\operatorname{IgE}^{+}$cells were never found in repeated experiments. Compared with recipient-derived naïve $\mathrm{B}$ cells, donor-derived IgG1 ${ }^{+}$ and $\operatorname{IgM} / \mathrm{D}^{+} \mathrm{B}$ cells were larger and expressed higher levels of B7.1, CD21, CD19 and MHC class-II. Although the iGB cells expressed high levels of the GC B-cell markers Fas and GL7, after transfer, they expressed only moderate levels of Fas and were GL7 negative (Fig. 2b,c). Additionally, they were CD38 ${ }^{\text {high }}$ and CD62L ${ }^{\text {int }}$, markers that are both low on GC as well as iGB cells. The donor-derived cells had lower CD23 levels than naïve B cells, and little of the CD138 and CD43 PC markers (Fig. 2b). These results indicate that a significant number of the transferred iGB cells survived for long periods in the spleen of the recipient mice and acquired the phenotype of $\mathrm{B}_{\text {mem }}$ cells, which were designated induced memory $\mathrm{B}$ (iMB) cells.
Generation of iMB cells is independent of antigenic stimulation. $\mathrm{B}_{\text {mem }}$ cells are maintained with no or very slow cycling ${ }^{39}$, so we asked whether the iMB cells are cycling in vivo. BrdU-pulsed iGB cells were transferred into irradiated mice and their kinetics were analysed. At the time of transfer, $73 \%$ of $\mathrm{IgG1}^{+}$cells were $\mathrm{BrdU}^{+}$ and after two weeks $\sim 30 \%$ of the cells were $\mathrm{BrdU}^{+}$, a percentage that remained largely unchanged even at 8 weeks after the transfer (Fig. 3a,b). These data indicate that the iMB cells became quiescent during the 6 weeks after the point of 2 weeks post-transfer, a time by which none of the donor B cells had the GC phenotype but exhibited the $B_{\text {mem }}$ phenotype. These results indicate that, after the $B_{m e m}$ phenotype is established, IgG1 ${ }^{+}$iMB cells are maintained with very little, if any, cycling in vivo. Very similar results were observed with IgM/D ${ }^{+}$iMB cells (Supplementary Fig. S2).

We examined whether BCR signalling is involved in the generation of $\mathrm{iMB}$ cells in vivo and found that stimulation of iGB cells with anti- $\mu \mathrm{H} \mathrm{F}\left(\mathrm{ab}^{\prime}\right)_{2} \mathrm{Ab}$ during the culture period did not affect the number of the resultant $\operatorname{IgG} 1^{+}$or $\operatorname{IgM} / \mathrm{D}^{+} \mathrm{iMB}$ cells in the recipient spleens (Fig. 3c; Supplementary Fig. S2). We next 
a
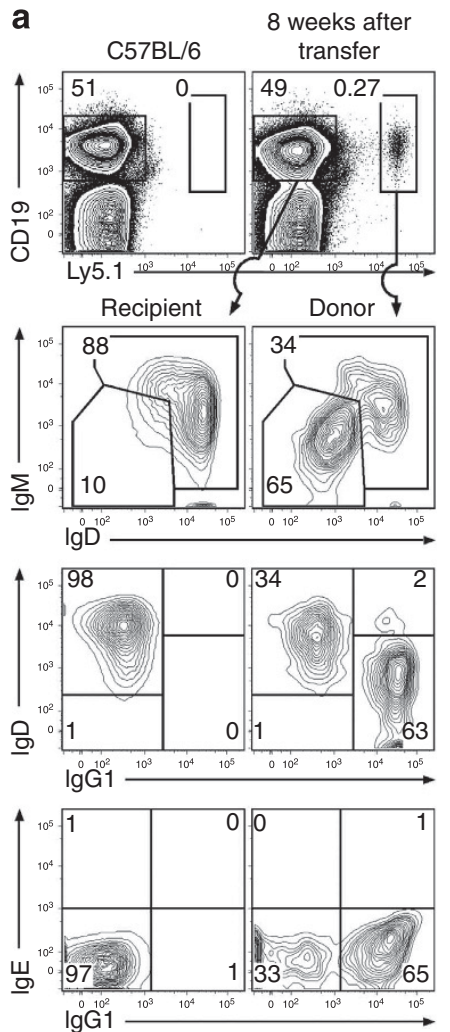

b

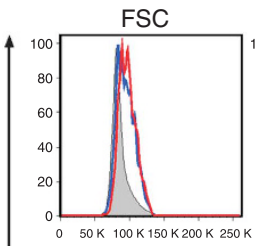

CD19

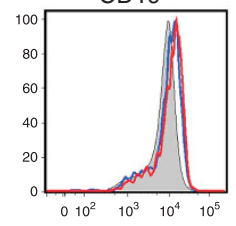

GL7

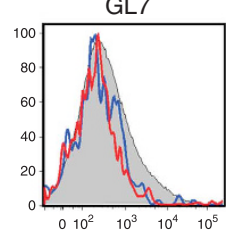

CD23

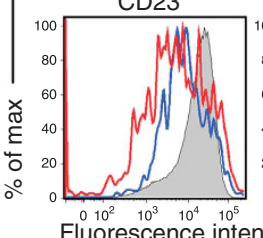

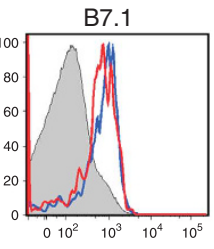

$\mathrm{MHCII}$

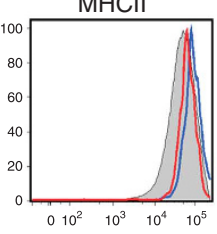

CD38

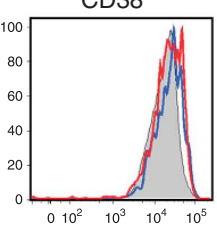

CD138

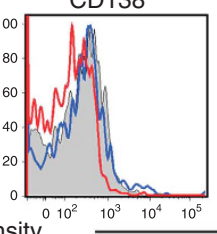

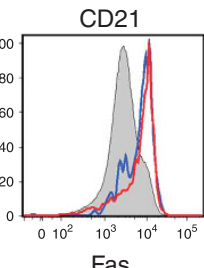

Fas

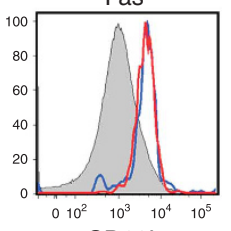

CD62L

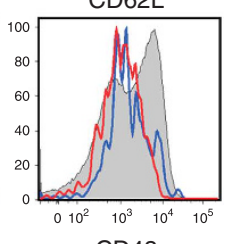

CD43

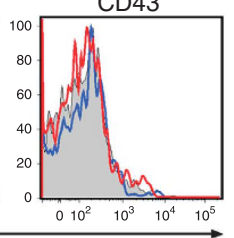

c
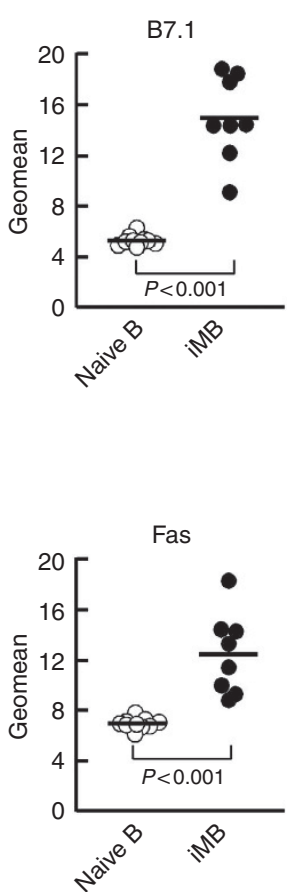

Figure $\mathbf{2}$ | iGB cells differentiate into cells with a $\mathbf{B}_{\text {mem }}$ phenotype in recipient mice. (a) Day 4 iGB cells derived from C57BL/6 Ly5.1+ congenic mice $\left(2 \times 10^{7}\right)$ were purified and transferred i.v. into $\gamma$-irradiated $(6.5 \mathrm{~Gy}) \mathrm{C} 57 \mathrm{BL} / 6$ mice (Ly5.2 ${ }^{+}$). Eight weeks later, pooled spleens of four recipients and a spleen of an untreated C57BL/6 mouse were stained with the indicated antibodies and analysed by flow cytometry. Ly $5.1^{+}$CD19+ ${ }^{+}$donor and Ly5.1- CD19+ recipient $B$ cells were gated as indicated and analysed for their BCR isotypes. (b) Expression of the indicated markers on the donor lgG1+ $\left(\mathrm{Ly} 5.1^{+} \mathrm{CD} 19^{+}\right.$

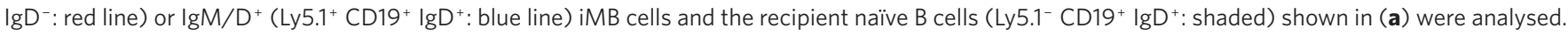
Anti-mouse lgG1 Ab was not used because it often cross-reacts with rat antibodies. The data shown are representative of three independent experiments performed with three to four mice per group. (c) Expression levels of B7.1 and Fas on Ly5.1+ ${ }^{+}$MB cells (closed circles; $n=8$ ) or Ly5.1- naïve B cells from the recipient and two normal C57BL/6 mice (open circles; $n=10$ ). Plotted are geometric means (geomean) of the fluorescence intensity of the cells from individual mice with the average value of each group of mice indicated by a horizontal line.

generated iGB cells from two mouse lines, each expressing knockin/transgenic BCR specific for a known external antigen: Hy10 (refs 40,41 ), which is specific for hen egg lysozyme (HEL), and B1-8 $\mathrm{ki}^{42}$, which is specific for (4-hydroxy-3-nitrophenyl) acetyl (NP) when co-expressing $\lambda \mathrm{L}$ chains. The iGB cells derived from these mice, about half of which were Ag specific, (Fig. 3d,f), gave rise to iMB cells in the recipients at a frequency similar to the non-transgenic B cells, and, moreover, these iMB cells could persist as long as 3 months without antigen. The iMB cells in both cases exhibited a $B_{\text {mem }}$ phenotype (B7.1 $1^{+}$and CD38 $8^{\text {high }}$ ) and maintained the same proportion of $\mathrm{IgG} 1^{+}$antigen-binding $\mathrm{B}$ cells as in the pre-transfer cells (Fig. 3e,g). These data indicate that antigenic stimulation is not required for generation of iMB cells.

iMB cells function as $\mathbf{B}_{\text {mem }}$ cells. To investigate whether iMB cells rapidly respond to $\mathrm{T}$-dependent (TD) adjuvant-free antigen similarly to physiological $\mathrm{B}_{\text {mem }}$ cells, mice containing Hy10-derived iMB cells, generated as in Figure $3 e$, were injected with splenocytes containing memory $\mathrm{T}$ cells primed with ovalbumin (OVA) and then immunized i.v. with a soluble OVA-HEL conjugate. Five days later, spleens of the recipient mice were analysed by flow cytometry (Fig. 4a). The unimmunized control mice contained a single population of iMB cells that had high levels of HEL binding (HEL ${ }^{\text {high }}$, whereas the immunized mice contained another population of donor-derived cells that bound less HEL (HEL $\left.{ }^{\text {low }}\right)$. HEL ${ }^{\text {high }}$
iMB cells in both mice exhibited the $B_{\text {mem }}$ phenotype whereas $\mathrm{HEL}^{\text {low }}$ cells in the immunized mice were larger and mostly exhibited a plasmablast phenotype: $\mathrm{CD} 138^{+}, \mathrm{CD} 43^{+}, \mathrm{CD} 19^{\text {low }}$, surface IgG $1^{\text {low, }}$ and $\mathrm{CD} 38^{\text {low. }}$. Using immunofluorescence microscopy, we showed that, in unimmunized mice, $\mathrm{HEL}^{+}{ }^{\mathrm{iMB}}$ cells were localized in the perifollicular regions and mostly scattered along the MAdCAM- $1^{+}$marginal sinus (Fig. $4 \mathrm{~b}-\mathrm{d}$ ). In the immunized mice, most of the $\mathrm{HEL}^{+} \mathrm{IgG1}^{+}$cells were localized in the extrafollicular regions, in the red pulp or at the junction of the T-cell zone and the red pulp (Fig. $4 \mathrm{e}-\mathrm{g}$ ). The $\mathrm{HEL}^{+}$iMB cells did not form GCs in the follicles, as has also been reported for bona fide $\mathrm{B}_{\text {mem }}$ cells ${ }^{43}$.

To examine $\mathrm{Ab}$ production by iMB cells, splenic B cells containing $\mathrm{HEL}^{+} \mathrm{iMB}$ cells shown in Figure 3e or naïve follicular or marginal zone (MZ) B cells from Hy10 mice were co-transferred with splenocytes from OVA-primed mice into secondary recipient mice. On immunization i.v. with soluble OVA-HEL, the mice that received $\mathrm{HEL}^{+} \mathrm{iMB}$ cells produced anti-HEL IgG1 Ab, which was detectable as early as day 3.5 and peaked by day 7 after immunization, but mice that received naïve follicular or MZ B cells or no $\mathrm{HEL}^{+}$ $B$ cells did not respond (Fig. 4h). Similar results were observed in the model of $\mathrm{NP}^{+}$iMB cells and carrier-primed CD4 ${ }^{+} \mathrm{T}$ cells (Fig. 3g; Supplementary Fig. S3). When Hy10-derived IgG1 ${ }^{+}$and $\operatorname{IgM} / \mathrm{D}^{+}$ iMB cells were separately transferred into mice with OVA-primed T cells, both types of iMB cells responded to immunization with soluble OVA-HEL, as shown by expansion, differentiation into CD138+ 
a

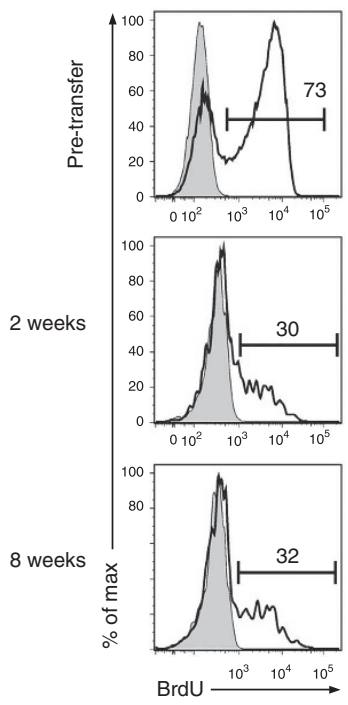

b

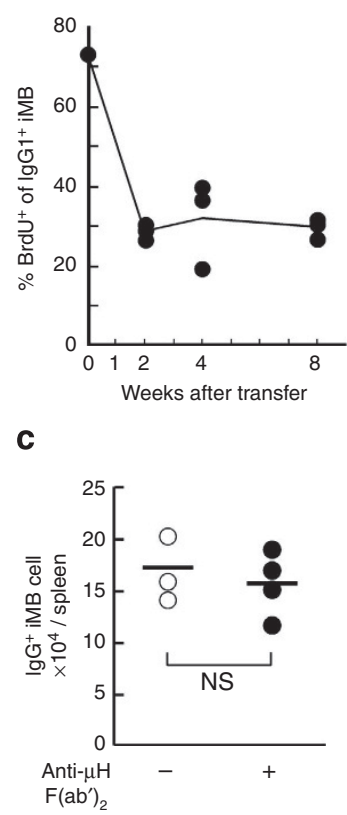

d
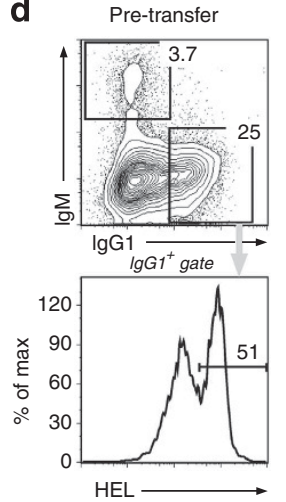

f
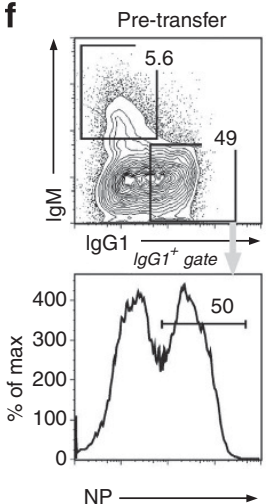
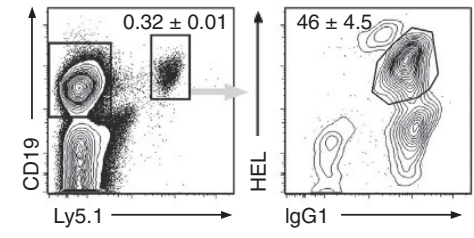
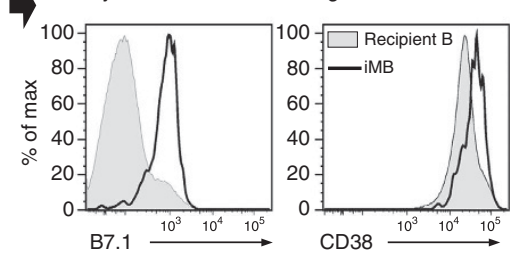

g
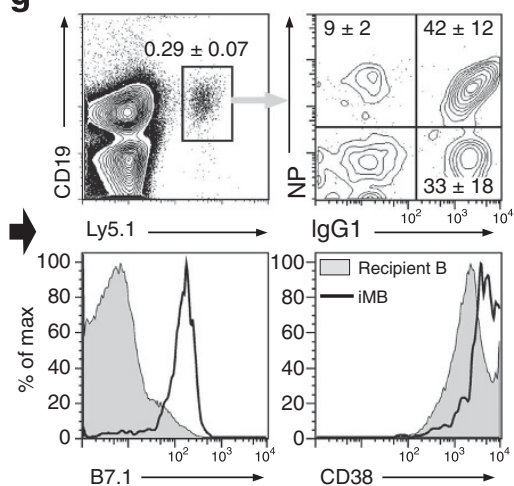

Figure 3 | Generation and maintenance of iMB cells are independent of antigenic stimulation. (a,b) In vivo BrdU-pulse chase analysis of iMB cells. Ly5.1+ iGB cells were pulsed with BrdU or not for the last $7 \mathrm{~h}$ of a 4-day culture and then transferred into irradiated (6.5 Gy) C57BL/6 mice. BrdU content was analysed by flow cytometry in the IgG1 ${ }^{+}$pre-transfer iGB cells $(n=1)$ and $\operatorname{lgG} 1^{+}$Ly $5.1^{+} \mathrm{CD} 19^{+}$donor iMB cells in the spleens of the recipient mice 2,4 , or 8 weeks after transfer ( $n=3$ for each time point). (a) Representative data with numbers indicating percentages of BrdU-containing pre-transfer and $\mathrm{iMB}$ cells. Unpulsed iGB and iMB cells are shown as controls (shaded). (b) Kinetics of the percentage of BrdU ${ }^{+}$iMB cells in individual recipients. The value of pre-transfer cells is plotted at 0 -week. Data shown are representative of two independent experiments. (c) The effect of initial BCR stimulation in vitro on iMB generation in vivo. Mice had received Ly5.1 ${ }^{+}$iGB cells cultured with (closed circles: $n=4$ ) or without (open circles: $n=3$ ) extra anti- $\mu \mathrm{H}$ $\mathrm{F}\left(\mathrm{ab}^{\prime}\right)_{2}\left(1 \mu \mathrm{g} \mathrm{ml} \mathrm{l}^{-1}\right) 62$ days before. Absolute numbers, with an average in each group (horizontal bars), of $\operatorname{lgG} 1^{+} \mathrm{CD} 19^{+} \mathrm{Ly} 5.1^{+}$iMB cells in each spleen of individual recipient mice are plotted. The cell number is estimated on the basis of the cell frequency determined by flow cytometry and total cell number. n.s.: not significant. Similarly, no effect of BCR stimulation was observed in the recipients 20 days after transfer in an independent experiment. (d-g) Day $4 \mathrm{iGB}$ cells derived from total splenic B cells from Hy10 Ly5.1+ mice (d) or those enriched for NP ${ }^{+}$cells from B1-8 ki Ly5.1 ${ }^{+}$mice (f) were transferred into irradiated C57BL/6 mice. The frequency of $\mid g M^{+}$and $\operatorname{lgG} 1^{+}$cells $(\mathbf{d}, \mathbf{f})$, and of $\operatorname{lgG} 1^{+}$cells binding to $\mathrm{HEL}(\mathbf{d})$ or $\mathrm{NP}(\mathbf{f})$ antigens in the pre-transfer iGB cells are shown. For unknown reasons, the iGB cells with a knock-in IgH gene tend to undergo isotype switching more rapidly and to express lower BCR levels than wild-type iGB cells. (e) The frequencies of $\mathrm{Ly} 5.1^{+} \mathrm{CD} 19^{+}$iMB cells in the pooled spleen cells from four recipient mice 37 days after transfer with the cells shown in (d), and profiles of these iMB cells illustrating antigen-binding and IgG1 expression, B7.1 expression, and CD38 expression, are shown. (g) The frequency of iMB cells in the pooled spleen cells from four recipient mice 91 days after transfer with the cells shown in (f), and the same profiles as above, are shown. Data are representative of three or more experiments each performed with two-to-five recipient mice.

plasmablasts, and production of anti-HEL IgG1 at equivalent levels (Supplementary Fig. S4).

These results indicate that, in the presence of memory $\mathrm{T}_{\mathrm{H}}$ cells, iMB cells are able to differentiate rapidly into plasmablasts and begin to produce $\mathrm{Ab}$ after immunization with soluble, adjuvantfree TD antigen. Taken together with the other iMB cell features described above, we conclude that iMB cells are phenotypically and functionally equivalent to physiological $\mathrm{B}_{\mathrm{mem}}$ cells.

IL-21 boosts proliferation of IL-4-induced iGB cells. IL-21 is required for maximal expansion of GC B cells ${ }^{9-12}$, and facilitates proliferation of BCR-/CD40-stimulated B cells ${ }^{14,44}$. Primary culture with IL-21 on 40LB feeder cells for 4 days resulted in 25 -fold less proliferation of B cells than in the cultures with IL-4, with less frequent or little switching to IgG1 and IgE, respectively. Despite the known synergistic effect of IL-4 and IL-21 for Ig production in $v i v o^{12}$, synergy in proliferation was not evident in the primary cultures (Supplementary Fig. S5). Next, iGB cells were first cultured with IL-4 and then subsequently cultured for 2 days either with IL-21 or IL-4 (Fig. 5a). As shown in Figure 5b, proliferation was greater in the secondary culture with IL-21 than with IL-4, and the IgG1 ${ }^{+}$cells dominated over $\operatorname{IgE}^{+}$cells, which was opposite the case in the parallel IL-4 culture (Fig. 5c). We designated iGB cells cultured with IL-4 as iGB-4, and with IL-21 in the secondary culture as iGB-21 cells. The iGB-21 cells maintained logarithmic proliferation in further culture with IL-21 until day 8 , expanding 10,000-fold (Fig. 5b). Whereas the frequency of $\mathrm{CD}_{138^{+}}$cells was increased only among the $\mathrm{IgE}^{+}$iGB4 cells, it was increased in both $\operatorname{IgE}^{+}$and $\mathrm{IgG} 1^{+}$iGB-21 cells on day 8 (Fig. 5c), perhaps correlating with the preferential contribution of IL-4 and IL-21 to IgE and IgG1 antibody production, respectively, in TD immune responses ${ }^{12,45}$. Parallel culture of the iGB-21 cells on 40LB cells without cytokines from day 6 (iGB-m cells) resulted in a lower growth rate. The majority of the iGB-m cells as well as iGB-21 and iGB- 4 cells were CD138 ${ }^{-}$and exhibited a GC phenotype (Fas ${ }^{+}$ 


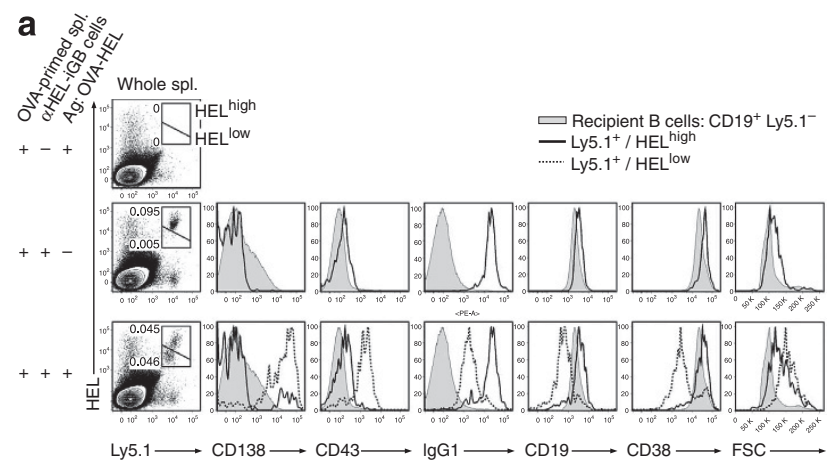

b

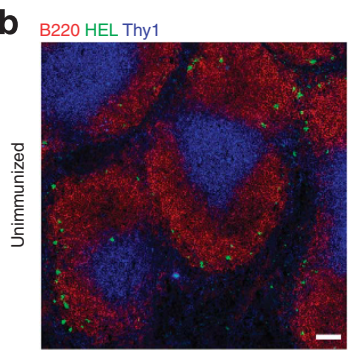

e B220 HEL Thy 1

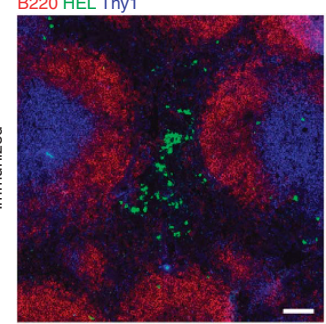

h

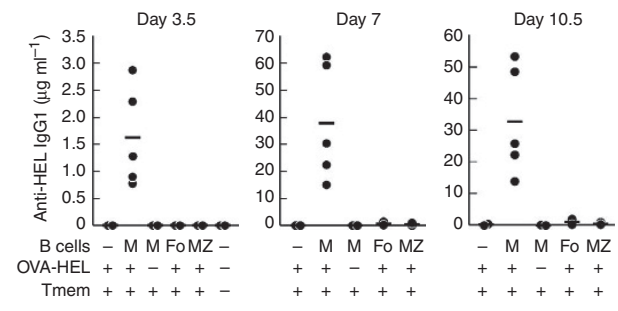

d

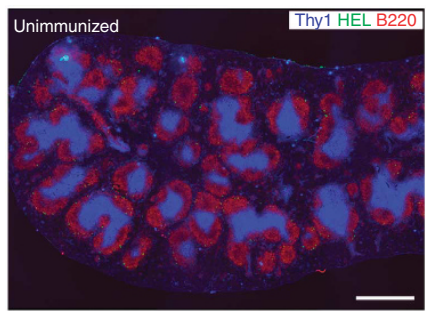

g

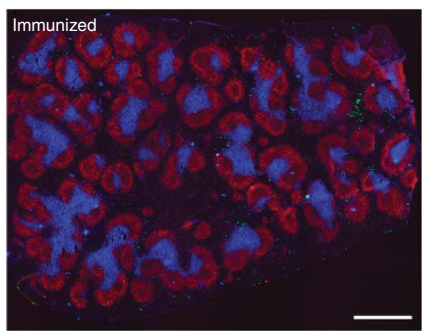

Figure 4 | iMB cells rapidly differentiate into plasma cells in vivo on immunization. (a) Control C57BL/6 mice ( $\alpha \mathrm{HEL}-\mathrm{iGB}$ cells: - ) or mice that received Hy10 Ly5.1 $1^{+}$iGB cells 50 days before ( $\alpha \mathrm{HEL}-\mathrm{iGB}$ cells: + ) were injected i.v. with $2 \times 10^{7}$ pooled splenocytes from mice primed with OVA with CFA 2 months before, and immunized i.v. with OVA-HEL (20 $\mu \mathrm{g})$ in PBS (Ag:OVA-HEL: +) or not (Ag:OVA-HEL: -) $12 \mathrm{~h}$ later. Five days after immunization, spleen cells were analysed by flow cytometry. Each histogram shows the staining profile of the indicated marker in Ly5.1 ${ }^{+} \mathrm{HEL}^{\text {high }}$ or HEL $\mathrm{L}^{\text {low }}$ donor B cells and $C D 19^{+}$Ly $5.1^{-}$recipient B cells defined in the dot plots. (b-g) Photomicrographs of sections of the same spleens of the mice receiving iGB cells in (a), unimmunized (b-d) or immunized (e-g). Sections were stained with anti-B220 (red), HEL (green), and anti-Thy1 (blue) (b,d,e,g); anti-MAdCAM-1 (red), HEL (green), and anti-B220 (blue) (c); or HEL (red), anti-lgG1 (green), and anti-B220 (blue) (f). Data are representative of three independent experiments. Magnification is $\times 20(\mathbf{b}, \mathbf{c}, \mathbf{e}, \mathbf{f})$ or $\times 4(\mathbf{d}, \mathbf{g})$. Bars: $100 \mu \mathrm{m}(\mathbf{b}, \mathbf{c}, \mathbf{e}, \mathbf{f})$ or $1 \mathrm{~mm}(\mathbf{d}, \mathbf{g})$. (d, g) show low power views of the images in $(\mathbf{b})$ and $(\mathbf{e})$, respectively. (h) Splenocytes $\left(2 \times 10^{7}\right)$ from OVA-primed mice $\left(T_{\text {mem }}\right)$ were transferred i.v. into non-irradiated C57BL/6 mice with the HEL ${ }^{+}$iMB cellcontaining splenic B cells shown in Figure $3 \mathrm{~d}(\mathrm{M})(n=5)$, with sorted HEL+ follicular B cells (Fo) $(n=2)$ or MZ B cells $(M Z)(n=2)$ from naïve Hy10 mice, or without B-cells $(-)(n=2)$. All donor B cells contained $1.2 \times 10^{5} \mathrm{HEL}^{+}$B cells. After i.v. immunization with $(+)$or without $(-) 20 \mu g$ OVA-HEL, serum titres of anti-HEL IgG1 in individual mice were determined on the indicated days. Horizontal bars indicate the average value in each group. Data shown are representative of three independent experiments performed with two-to-five mice per group.

and $\mathrm{GL7}^{+}$) (Fig. 5c). These results indicate that IL-21 promotes extensive proliferation and partial differentiation towards PC of iGB cells in vitro.

Compared with the CD138- iGB-4 cells, expression of Bcl-6 and $\mathrm{Bcl}-\mathrm{xL}$ proteins was lower in the CD138- $\mathrm{iGB}-21$ cells, but higher than in ex vivo GC B cells (Fig. 1g). The messenger RNA levels of Aicda, which encodes activation-induced cytidine deaminase (AID) that is indispensable for CSR and SHM, was $\sim 4$ - or $\sim 10$-fold lower in the IgG1 ${ }^{+}$iGB- 4 or iGB-21 cells, respectively, than the GC B cells (Supplementary Fig. S6). In relation to this diminished level of Aicda expression, the iGB-21 cells derived from B1-8 ki B cells had no detectable $\mathrm{V}_{\mathrm{H}}$ gene mutations on day 8 of the culture with or without continuous BCR stimulation by anti- $\kappa$ antibody. Forced expression of AID in the iGB cells (Supplementary Methods) resulted in the accumulation of $\mathrm{V}_{\mathrm{H}}$ mutations. The mutation rate was estimated as $1.5 \times 10^{-4}$ per base pair per generation (roughly estimated as 8 generations after AID expression), which was within the range reported in physiological GC B cells ${ }^{46}$ (Supplementary Fig. S6). The mutations consisted of C-to-T/G-to-A transitions, possibly generated through AID-mediated C-deamination, as well as other substitutions and a deletion, typical of the mutations normally seen in immunized animals, suggesting that iGB cells possess all of the essential SHM machinery operative in physiological GC B cells ${ }^{47}$. This result also suggests that a higher level of AID is required for SHM than for CSR.

IL-21 reversibly suppresses iMB cell development from iGB cells. To examine the effect of IL-21 on the in vivo differentiation of iGB cells into iMB cells, we transferred an equal number of $\operatorname{IgG1}^{+}$ CD138- ${ }^{-}$Ly $5.1^{+}$, iGB-4, iGB-21 or iGB-m cells into C57BL/6 mice and analysed them 30 days later. Ly5.1 ${ }^{+}$iMB cells were detected in the spleen, lymph node, peritoneal cavity(PerC), and blood from the iGB-4 cell (iMB-4) and iGB-m cell (iMB-m) recipients (Fig. 6a). Most of the iMB cells were $\operatorname{IgG} 1^{+}$but never $\operatorname{IgE}^{+}$(Supplementary 

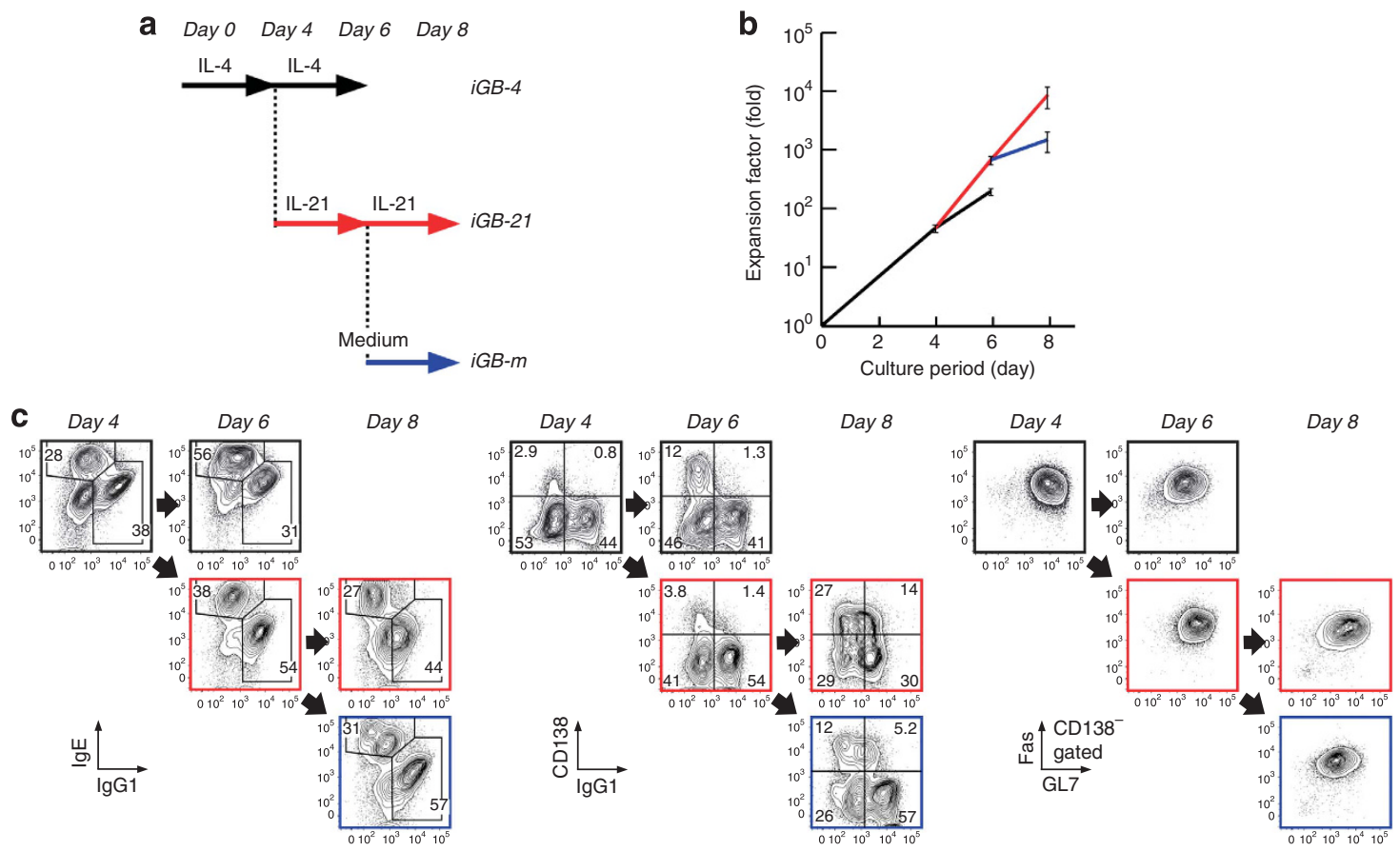

Figure 5 | IL-21 accelerates proliferation of iGB cells and partial differentiation of IgG1+ iGB cells into plasmablasts. (a) Schematic diagram of the different iGB cell cultures performed in (b,c). In vitro generated iGB cells were successively cultured on 40 LB cells with IL-4 (iGB-4; black), or with IL-21 replacing IL-4 on day 4 (iGB-21; red). Additionally, the iGB-21 cells were deprived of IL-21 on day 6 and successively cultured on 40LB without added cytokines (iGB-m; blue). (b) Cumulative fold increase on day 4, 6, and 8, of B cells cultured as described in (a) was estimated from three independent experiments and represented as in Figure $1 \mathrm{~b}$. Colours of the lines indicate the culture conditions described in (a). (c) Flow cytometric analysis of the expression of Ig isotypes and CD138 on iGB cells, and of Fas and GL7 on CD138 ${ }^{-}$gated cells, cultured as in (a). Colours of the boxes indicate the culture conditions described in (a). Numbers indicate the percentages of cells in the quadrants or windows. Data shown are representative of five independent experiments.

Fig. S7). Absolute numbers of iMB-4 and iMB-m cells in the recipient spleens were $2-5 \times 10^{5}$ and $0.5-1 \times 10^{5}$, respectively, $30-50$ days after the transfer, and gradually decreased thereafter (Fig. 6b). In striking contrast, essentially no iMB cells were detected in the mice adoptively transferred with iGB-21 cells (Fig. 6a,b).

The iMB-m cells had the same memory phenotype as iMB-4 cells (Fig. 6c,d; Supplementary Fig. S7). Both types of iMB cells in the spleen, but not in lymph node, exhibited a phenotype similar to MZ B cells in terms of CD21 versus CD23 expression (Fig. 6c), and expressed a low level of IL-21R, as reported for human $\mathrm{B}_{\mathrm{mem}}$ cells ${ }^{48}$ (Fig. 6d; Supplementary Fig. S7). The iMB-m cells were comparable to iMB-4 cells in their function to respond to soluble Ag in the secondary recipient mice, whereas $B$ cells from the iGB-21recipients, which contain no discernable iMB cells, failed to respond (Fig. 6e). These results indicate that the ability of iGB cells to differentiate into functional iMB cells is inhibited by IL-21 in vitro, and also that this inhibition is reversible.

IL-21 facilitates LLPC development from iGB cells. LLPCs are generated during the late phase of the GC reaction'; therefore, we examined whether iGB cells differentiate into LLPCs by estimating the number of $\mathrm{Ab}$-forming cells (AFC) in the bone marrow (BM) of mice that received $\mathrm{IgG}^{+}{ }^{+} \mathrm{NP}^{+} \mathrm{CD} 138^{-}$iGB cells derived from $\mathrm{B} 1-8$ ki mice. Anti-NP IgG1-secreting AFCs were detected in mice that received iGB- 21 cells 4 weeks previously, but very few were seen in mice that received iGB-4 or iGB-m cells (Fig. 7a). Similarly, AFCs in spleen were only found in the mice receiving iGB-21 cells. The iGB-21-derived LLPCs were undetectable by flow cytometry, probably because of their scarcity $(\sim 0.005 \%$ as estimated from the data in Fig. 7a) and also because Ly5.1 (CD45.1) is downregulated on PCs ${ }^{49}$.
Serum anti-NP IgG1 titres correlated with the AFC numbers in the different recipient mice. The $\mathrm{IgG1}{ }^{+} \mathrm{CD} 138^{-}$iGB-21 cells appeared to be committed to the plasma-cell lineage because Blimp1 mRNA expression level was relatively high in these cells, whereas it was very low in the IgG1 ${ }^{+}$CD138- iGB-4 or iGB-m cells, each cultured for the same time period as the iGB-21 cells (Fig. 7b). Moreover, nearly $40 \%$ of the IgG1 ${ }^{+}$CD138- iGB-21 cells on day 6 , and even more on day 8 , were secreting anti-NP IgG1, whereas essentially none of the IgG1 ${ }^{+}$CD138- iGB-4 (on day 6) or iGB-m (on day 8) cells were secreting (Fig. 7c). These data indicate that secondary culture with IL-21 induces differentiation of iGB cells into CD138- AFCs, presumably the early precursors of LLPCs, but the CD138- AFCs disappear during the subsequent culture without IL-21. As expected, CD $138^{+}$iGB cells arising in any culture condition expressed high levels of Blimp1 mRNA and were mostly AFCs (Fig. 7b,c).

\section{Discussion}

We have described a novel B-cell culture system in which naïve B cells proliferate robustly, undergo isotype switching, and acquire a cell surface phenotype equivalent to GC B cells, termed iGB cells. After primary culture with IL-4 (iGB-4 cells), the cells develop into memory-like iMB cells in vivo, whereas after subsequent culture with IL-21 (iGB-21 cells) develop into LLPC-like cells in vivo. Bcl-6 and $\mathrm{Bcl}-\mathrm{xL}$ proteins, which are among the signature proteins of physiological GC B cells ${ }^{38}$, are abundantly expressed in $\mathrm{iGB}-4$ and $\mathrm{iGB}-21$ cells. It was recently reported that human peripheral memory B cells transduced with Bcl- 6 and Bcl-xL could be propagated as GC-like B cells for a long period on feeder cells expressing CD40L and supplemented with IL-21 (ref. 50). Thus, the high-level expression of Bcl-6 and Bcl-xL, together with signalling 

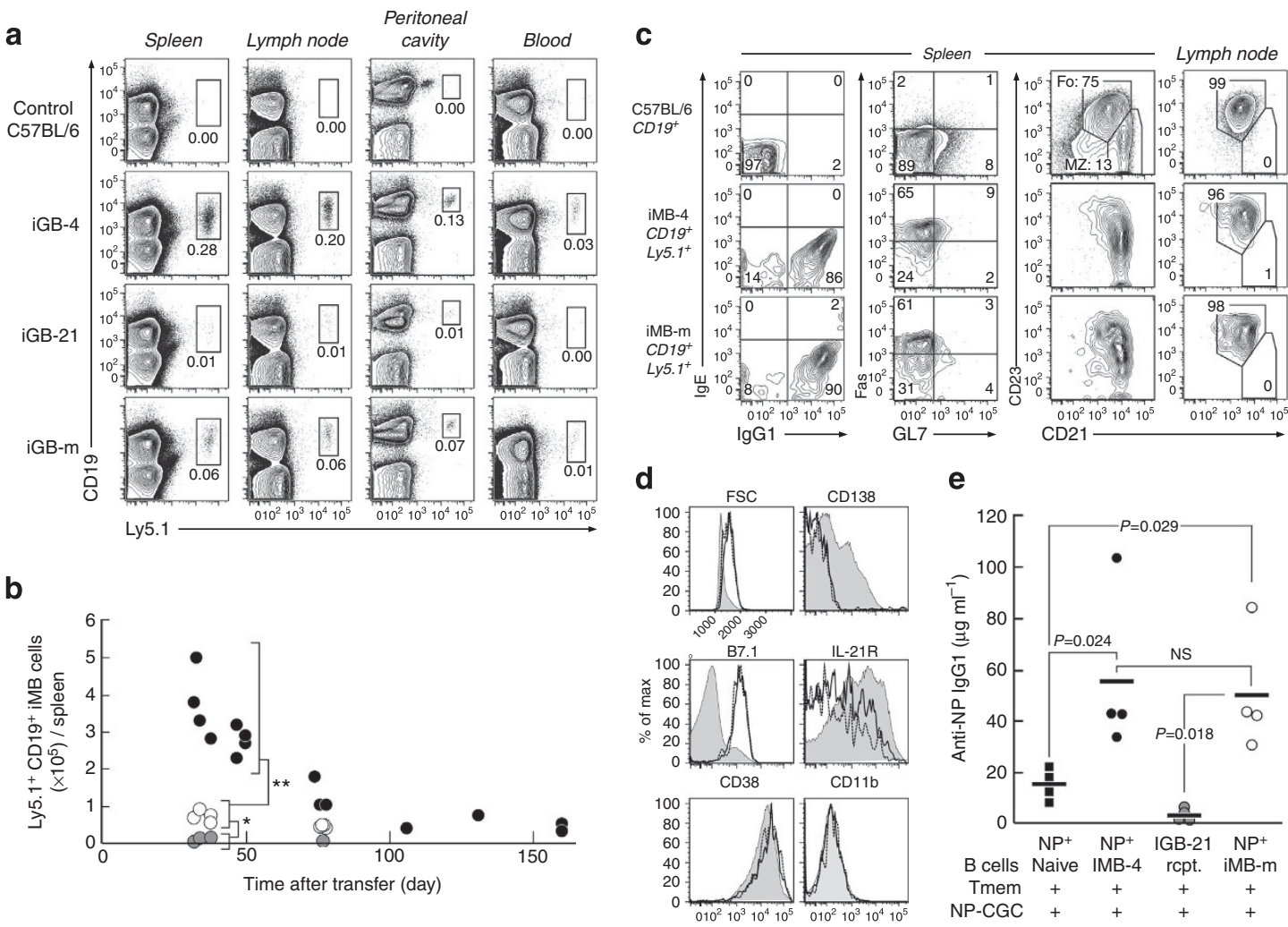

Figure 6 | IL-21 reversibly suppresses the differentiation of iGB cells into iMB cells. (a) Flow cytometric analysis for CD19/Ly5.1 expression on the cells from indicated tissues of untreated C57BL/6 mice (B6) or recipient mice that received iGB-4, iGB-21, or iGB-m cells, each devoid of CD138- cells and containing $1 \times 10^{7} \operatorname{lgG} 1^{+}$cells, 30 days before. The numbers indicate the percentage of $\mathrm{iMB}$ cells defined by each window. (b) The numbers of splenic iMB4 (closed circles: $n=15$ ), iMB-21 (shaded circles: $n=4$ ) or iMB-m (open circles: $n=8$ ) cells were estimated by the analysis as shown in (a) in individual recipient mice on the indicated days. Difference is significant between the iMB-4 and iMB-m cells ( $\left.{ }^{\star \star} P<0.000006\right)$ and between iMB-m and iMB-21 cells $\left({ }^{\star} P<0.01\right)$ from the recipient mice analysed by day 50 after transfer. Data from 11 experiments were combined. (c) iMB-4 and iMB-m cells $\left(C D 19{ }^{+}\right.$Ly5.1 $\left.{ }^{+}\right)$ in the spleens of mice generated as in (a) and in control CD19+ $B$ cells from untreated B6 mice were analysed for expression of the indicated markers. The numbers indicate the percentage of cells defined by each window. (d) Expression of the indicated markers on the splenic iMB-4 (thick line), iMB$\mathrm{m}$ (dotted line) or B6 naïve (shaded) B cells. (c,d) Data shown are representative of three or more independent experiments. (e) Splenic B cells of the recipient mice containing $8 \times 10^{3} \mathrm{NP}^{+}$iMB-4 or iMB-m cells, or those of a B1-8 ki mouse containing $8 \times 10^{3} \mathrm{NP}^{+}$naïve $\mathrm{B}$ cells (the numbers were estimated on the basis of their frequency in flow cytometric analyses), each supplemented with naïve B cells of untreated B6 mice to make the total B-cell number $2.4 \times 10^{7}$, or splenic B cells $\left(2.4 \times 10^{7}\right)$ of the recipient having received iGB-21 cells ('iGB-21 rcpt.'), were co-transferred with CGG-primed CD4 ${ }^{+} T$ cells ( $\mathrm{T}_{\text {mem }}$ ) into $\gamma$-irradiated $B 6$ mice. Seven days after i.v. immunization with $\mathrm{NP}_{40}-\mathrm{CGG}$, serum titres of anti-NP IgG1 in individual mice $(n=4$ for each group) were measured. Data shown are representative from two independent experiments.

through CD40 and the IL-21 receptor, seem to be required for the extensive B-cell proliferation in vitro. In our system, initiating the culture with IL-21 resulted in a poor proliferation of the iGB cells, suggesting that the primary IL-4-receptor signalling may be required for sufficient upregulation of $\mathrm{Bcl}-6$ and $\mathrm{Bcl}-\mathrm{xL}$ proteins in naïve B cells.

Somewhat unexpectedly, the level of $B c l-6$ mRNA was low in iGB cells compared with ex vivo GC B cells (Supplementary Figs S6 and S8). However, it is well known that Bcl-6 mRNA and protein levels can be discordant. For example, resting and GC B cells have similar $\mathrm{Bcl}-6$ message levels, yet Bcl-6 protein levels are much higher in the GC B cells, suggesting an important role for post-transcriptional regulatory mechanisms ${ }^{51}$. CD40 signalling has been shown to suppress $\mathrm{Bcl}-6$ transcription via Irf4 (refs 51,52). As both iGB-4 and iGB-21 cells express abundant Irf4 protein (Fig. 1g), the continuous stimulation through CD40 in the culture may cause the suppression of $\mathrm{Bcl}-6$ gene expression. Thus, $\mathrm{Bcl}-6$ protein must be induced in the initial phase of the primary culture with IL-4 and maintained with only slow decay during the secondary culture with IL-21 (Fig. 1g). The levels of Bcl-6 protein may reach an insufficient level around day 10, when the iGB-21 cells stop proliferating. This assumption is supported by our preliminary data demonstrating that retroviral transduction of Bcl-6 endowed the iGB-21 cells with unlimited growth capacity in the presence of IL-21 and 40LB feeder cells.

Despite their GC-like phenotype and high proliferative potency, the iGB-21 cells failed to mutate their Ig genes, a feature at least partly due to insufficient expression of AID for unknown reasons. The decreasing level of AID mRNA expression in iGB-21 cells inversely correlated with the increasing level of Blimp-1 mRNA (Supplementary Fig. S6 and Fig. 7b), and therefore Blimp-1 might downregulate the AID gene expression as proposed previously ${ }^{53}$.

One prominent feature of this system is that the developmental fate of the iGB cells, either becoming $B_{\text {mem }}$ cells or LLPCs, can be directed by cytokines. IL- 4 directs the cells to the iMB cell fate, but not into LLPCs, in vivo. The iMB cells exhibit a uniform $\mathrm{B}_{\text {mem }}$ phenotype, persist in vivo for months without cell division, and rapidly differentiate into PCs in the red pulp of the spleen in response to soluble TD antigen. These features confirm that the iMB cells are physiologically equivalent to $B_{\text {mem }}$ cells. Given this premise, it is noteworthy that iMB cells are induced in vitro and 

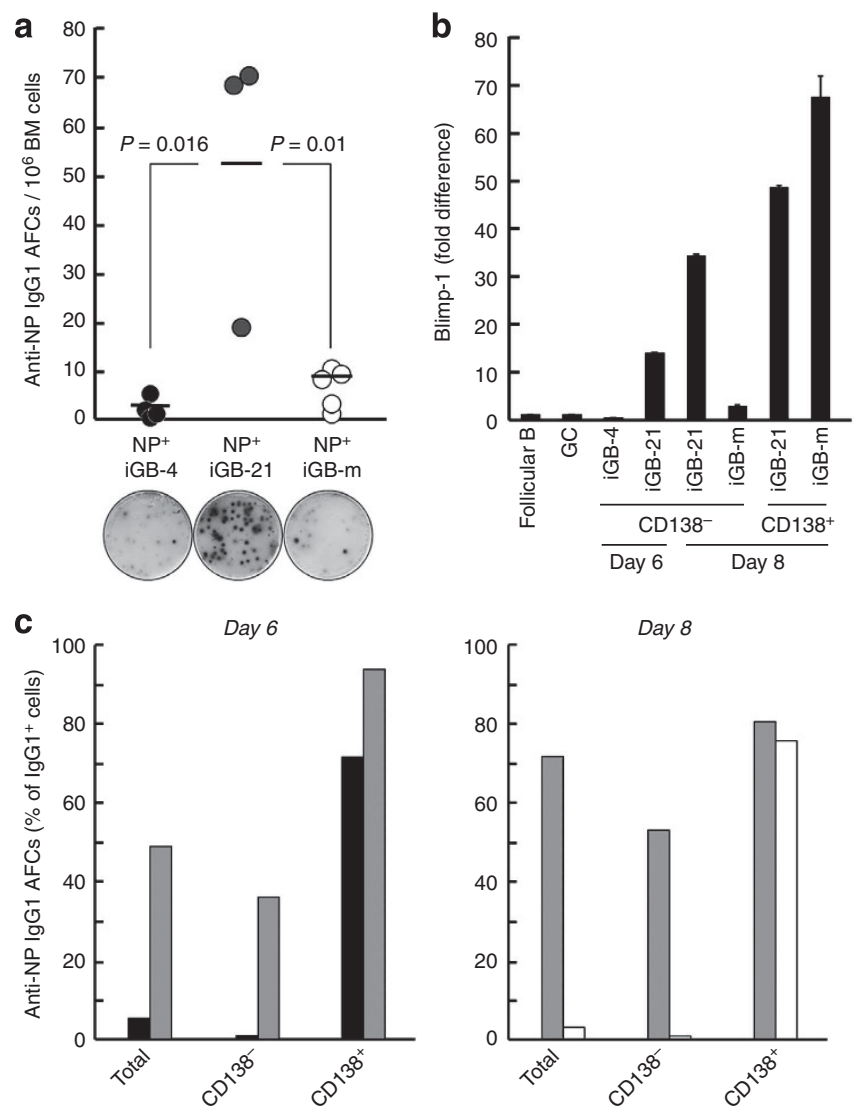

Figure 7 | IL-21 promotes differentiation of iGB cells towards plasma cells. (a) The frequency of NP-specific IgG1 AFCs among BM cells determined by ELISPOT assay. Shown is collective data with averages (bars) from five experiments using individual mice that had received iGB-4 $(n=4)$, iGB-21 $(n=3)$, or iGB-m $(n=5) \mathrm{CD}_{138^{-}} \operatorname{lgG} 1^{+} \mathrm{NP}^{+} \mathrm{iGB}$ cells derived from B1-8 ki mice 26-30 days before. Photographs depict representative ELISPOT wells seeded with $5 \times 10^{5}$ total BM cells. (b) Quantitative real-time PCR analysis of Blimp-1 transcripts in the following cells: splenic follicular B cells (CD43- $\left.{ }^{-} \mathrm{CD}_{138^{-}}, \mathrm{CD} 19^{+}, \mathrm{CD} 23^{\text {high }}, \mathrm{CD} 21^{+}\right)$sorted from unimmunized C57BL/6 mice, physiological GC B cells (CD43-', CD138-, IgD ${ }^{-}, \mathrm{CD}^{-} 9^{+}$, $\mathrm{Fas}^{+}, \mathrm{GL}^{+}$) sorted from $\mathrm{C} 57 \mathrm{BL} / 6$ mice immunized with BSA/CFA 3 weeks before, $C D 138^{-} \operatorname{lgG} 1^{+}$cells sorted from iGB-4, iGB-21 or iGB-m cells, and CD138 ${ }^{+}$cells from iGB-21 or iGB-m cells, each collected on the indicated day of the culture. Expression levels relative to that in follicular B cells (set as 1) are shown. Data is representative of two independent experiments. Error bars represent s.d. of triplicate samples. (c) $\mathrm{CD} 138^{+}$or $\mathrm{CD}^{-138^{-}}$and IgG $1^{+}$cells were purified by MACS from iGB- 4 and iGB-21 cells on day 6 or iGB-21 and iGB-m cells on day 8, and the frequency of anti-NP $\mid \mathrm{gG} 1^{+}$AFCs in these cells and pre-purified total $\lg \mathrm{G}^{+}$cells was determined by ELISPOT. iGB-4, iGB-21 and iGB-m cells are shown as black, grey and white bars, respectively. Data is representative of two independent experiments.

develop in vivo in the absence of antigenic stimulation through the BCR. This indicates that, apart from its function for Ag presentation to $T$ cells, BCR signalling per se is not necessary for GC B cells to become $B_{\text {mem }}$ cells, in agreement with studies showing a sufficient memory recall response in Btk- and BLNK-deficient mice ${ }^{54,55}$. It is also an intriguing finding that no specific GC microenvironment is required for $\mathrm{B}_{\text {mem }}$ cell development, because the recipient mice do not develop GC. This result is consistent with previous findings that premature $\mathrm{B}_{\mathrm{mem}}$ cells can be produced in mice in which germinal centre formation is inhibited by anti-ICOS $\mathrm{Ab}$ administration ${ }^{56}$ or Bcl-6 deficiency ${ }^{27}$.
$\mathrm{IgM} / \mathrm{D}^{+} \mathrm{iMB}$ cells possessing all the same features as $\operatorname{IgG} 1^{+} \mathrm{iMB}$ cells, except for the Ig isotype, are generated at the same time as IgG1 ${ }^{+}$iMB cells, and they may correspond to the $\operatorname{IgM}^{+} \mathrm{B}_{\text {mem }}$ cells first identified in the TD immune response of Bcl-6-deficient mice ${ }^{27}$. In contrast, $\mathrm{IgE}^{+} \mathrm{iMB}$ cells are not generated in vivo from $\mathrm{IgE}^{+} \mathrm{iGB}$ cells. Expression levels of BAFF-R, a receptor for a B-cell survival factor, cannot account for this deficiency, because they were almost equal between $\operatorname{IgE}^{+}$and $\operatorname{IgG1} 1^{+} \mathrm{iGB}-4$ cells (Supplementary Fig. S1). It seems more likely that $\operatorname{IgE}^{+} \mathrm{iGB}-4$ cells are committed to differentiation towards PCs, as Blimp-1 mRNA levels were tenfold higher in the $\mathrm{IgE}^{+}$iGB- 4 cells than in the IgG1 ${ }^{+}$iGB- 4 cells, among other GC-related mRNAs, although still much lower than in PCs (Supplementary Fig. S8). This idea is consistent with the fact that $\mathrm{IgE}^{+}$cells arising from $\mathrm{IgG}^{+} \mathrm{GC} \mathrm{B}$ cells swiftly become PCs and that $\mathrm{IgE}^{+} \mathrm{B}_{\text {mem }}$ cells are barely detectable in mice even after immunization to induce an optimal IgE response $\mathrm{s}^{5}$.

B-cell intrinsic IL-21 signalling is necessary for optimal GC formation and high-affinity IgG1-producing LLPC formation during TD immune responses ${ }^{9-12}$. Accordingly, we demonstrated here that IL-21 maintained the logarithmic proliferation of iGB cells after primary culture with IL-4 and that the CD138- fraction of the iGB-21 cells differentiated to LLPCs, but not to iMB cells, in vivo. Perhaps relevant to this observation, Tarlinton and colleagues reported accelerated formation of $\mathrm{B}_{\text {mem }}$ cells that had undergone less SHM and affinity maturation in IL-21- or IL-21R-deficient mice, suggesting premature development of $\mathrm{B}_{\text {mem }}$ cells in the GC reaction'. Collectively, these observations suggest that IL-21 normally suppresses the differentiation of GC B cells towards $B_{\text {mem }}$ cells and instead promotes their differentiation towards LLPCs. Notably, this suppression was reversible in at least a fraction of iGB cells, allowing iMB cell development from the iGB cells after IL-21 deprivation (iGB-m cells). This reversible suppression did not directly correlate to the expression level of BAFF-R on the iGB cells (Supplementary Fig. S1), although the evaluation of surface BAFF-R here might have been compromised owing to possible downregulation by continuous stimulation with BAFF produced from the feeder cells in the prolonged culture. Whatever the mechanism for the IL-21-mediated suppression, this finding suggests that the iGB cells maintain plasticity in their differentiation potential for at least a few days under IL-21 stimulation. If this were to apply to a physiological GC B cell reaction, IL-21 might afford GC B cells sufficient time to be diversified and selected before their fate is determined. After this period, positively selected GC B cells would differentiate into either LLPC or $\mathrm{B}_{\text {mem }}$ cells, depending on further availability of IL-21.

\section{Methods}

Preparation of feeder cells. Mouse CD40L complementary DNA cloned into a pApuro2 expression vector (a gift of Dr T. Kurosaki) was transfected into $\mathrm{BALB} / \mathrm{c} 3 \mathrm{~T} 3$ fibroblasts (clone A31, provided by RIKEN BRC) by lipofection and puromycin $\left(5 \mu \mathrm{g} \mathrm{ml}^{-1}\right)$-resistant stable clones ( $40 \mathrm{~L}$ cells) were subsequently selected. Mouse BAFF cDNA cloned into a pCA-neo, a T7-tag-deleted variant of the pCAT7-neo expression vector ${ }^{57}$, was transfected into the 40L or 3T3 cells and G418 $\left(2 \mathrm{mg} \mathrm{ml}^{-1}\right)$-resistant clones were subsequently selected. Among the $40 \mathrm{~L}$ transfectants, a clone on which naive B cells proliferated most extensively in the presence of IL- 4 was named 40LB and used for the rest of the experiments. All $\mathrm{BALB} / \mathrm{c} 3 \mathrm{~T} 3$-derived cells were mycoplasma-free as determined using the PCR Mycoplasma Detection Set (Takara).

Mice. C57BL/6 mice were purchased from Japan SLC. B1-8 IgH knock-in (B1-8 ki) mice ${ }^{42}$ were backcrossed to C57BL/6 or congenic C57BL/6-Ly5.1 strains. Hy10 (formerly called HyHEL10) mice carrying a $\mathrm{V}_{\mathrm{H}}$ knock-in (VDJ9 ki) allele and an Ig- $\mathrm{K}$ transgene $(\kappa 5 \mathrm{tg})^{40,41}$ were backcrossed to $\mathrm{C} 57 \mathrm{BL} / 6$ or the congenic $\mathrm{C} 57 \mathrm{BL} / 6$ Ly5.1 strains. All mice were maintained in the mouse facility under specific pathogen-free conditions. Mouse procedures were performed under protocols approved by the Animal Care and Use Committee of the Tokyo University of Science.

Isolation of cells. Single-cell suspensions from lymphoid tissues were depleted of RBCs in ammonium chloride lysis buffer and then incubated with 2.4G2 (BD Biosciences) to block FcyRII/III before staining with other Abs. Naïve B cells 
were purified from the spleens by 2-step negative sorting, first by an iMag system (BD Biosciences) using biotinylated monoclonal Abs (mAbs) against CD43 (BD Biosciences), CD4, CD8, DX5, Ter-119 (eBioscience), and avidin-particleDM (BD Biosciences) and then by passing of the unbound cells through a MACS LS column (Miltenyi Biotec), yielding B cells of $>97 \%$ purity. $\mathrm{NP}^{+} \mathrm{B}$ cells were enriched from the purified splenic B cells of B1-8 ki mice with a MACS system using $\mathrm{NP}_{7}$-BSA-biotin, yielding $\mathrm{NP}^{+}$cells of $>50 \%$ purity. About $50 \%$ of purified B cells from Hy10 Ly5.1 ${ }^{+}$mice were HEL-binding and used without further purification. Follicular and MZ B cells from Hy10 mice were sorted by FACSAria II (BD Biosciences) after staining with CD21-FITC, CD23-PE (BioLegend), and CD19-allophycocyanin(APC) (eBioscience). CD4 ${ }^{+} \mathrm{T}$ cells from mice immunized with alum-precipitated CGG were purified with an iMag system using biotinylated mAbs against CD45R/B220, CD8, and CD11b (eBioscience), yielding CD4 ${ }^{+}$T cells of $>70 \%$ purity. For removal of feeder and plasmablasts/plasma cells, single-cell suspensions from the iGB cultures were first incubated with $2.4 \mathrm{G} 2$ and then with biotinylated mAbs against $\mathrm{H}-2 \mathrm{Kd}$ and CD138 (BD Biosciences) at room temperature in MACS Buffer (0.5\% BSA, 2 mM EDTA in PBS), followed by incubation with avidin-particle-DM (BD Biosciences) and purification by an iMag system. Thus purified iGB cells were used for the adoptive transfer into irradiated recipient mice.

iGB cell culture. Typically, purified B cells $\left(5 \times 10^{5}\right.$ cells per dish) were cultured in a $10-\mathrm{cm}$ tissue culture dish (BD Falcon) in the presence of $40 \mathrm{LB}$ cells $\left(3 \times 10^{6}\right.$ cells per dish) that had been irradiated with $120 \mathrm{~Gy} \gamma$-ray in $40 \mathrm{ml}$ RPMI-1640 medium (Sigma) supplemented with $10 \%$ FCS, $5.5 \times 10^{-5} \mathrm{M} 2$-ME, $10 \mathrm{mM}$ HEPES, $1 \mathrm{mM}$

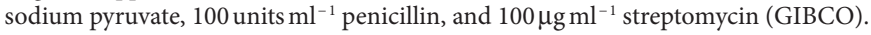
rIL-4 (1 ng ml ${ }^{-1}$; Peprotech) was added to the primary culture for 4 days, and on day 4 , the cells were re-plated onto a new feeder layer and cultured with rIL-21 (10 $\mathrm{ng} \mathrm{ml}^{-1}$; Peprotech) or IL-4 for 2-4 days (the secondary culture). In tertiary culture, cells were cultured on a new feeder layer without extra cytokines. Each time the cells were collected, the live B cell number was counted by the trypan blue dye exclusion method. Cells were cultured in a humidified atmosphere at $37^{\circ} \mathrm{C}$ with $5 \% \mathrm{CO}_{2}$.

Flow cytometry. Cells from lymphoid tissues depleted of RBCs were incubated with 2.4G2 and then stained with various combinations of the following Abs: FITC-, PE-, biotin-, PE-Cy7-, or APC-conjugated anti-mouse IgD, IgM, IgG1, and IgE (Southern Biotechnology), anti-mouse CD45.1 (BioLegend), anti-mouse CD5, CD19, CD21, CD23, CD38, CD40, CD45R/B220, CD80, CD138, CD154, CD11b, MHC-class II, PNA, and GL7 (eBioscience), anti-mouse CD43 (S7), CD95 (BD Biosciences), biotinylated-HEL, and biotinylated $\mathrm{NP}_{7}-\mathrm{BSA}$. The concentrations of the Abs used in this study are shown in the Supplementary Table S1. IL-21R was detected by a mouse IL-21-human C $\gamma 1$ fusion protein combined with PE-goatanti-human IgG. Cells were stained with propidium iodide, just before analysis, to eliminate dead cells in the data analyses. When the iGB cells were analysed, 40LB feeder cells were gated out based on FSC versus SSC. CFSE labelling was performed according to the manufacturer's instructions (Molecular Probes) in that freshly isolated splenic B cells $\left(1 \times 10^{7}\right.$ cells per $\left.\mathrm{ml}\right)$ were incubated with $2.5 \mu \mathrm{M}$ CFSE in $0.05 \% \mathrm{BSA}$ in $\mathrm{PBS}$ at $37^{\circ} \mathrm{C}$ for $10 \mathrm{~min}$ and then stained as above and used for the experiments. All samples were analysed using a FACSCalibur or FACSCanto II (BD Biosciences). The data were analysed using FlowJo (Tree Star).

BrdU labelling and detection. B cells were pulsed with $10 \mu \mathrm{M}$ BrdU for the last $1 \mathrm{~h}$ on day 3 or $7 \mathrm{~h}$ on day 4 of iGB culture. Single-cell suspensions were stained for surface markers as described above, washed with cold PBS, and resuspended in $0.5 \mathrm{ml}$ of cold $0.15 \mathrm{M} \mathrm{NaCl}$, to which $1.2 \mathrm{ml}$ of cold $95 \%$ ethanol was added dropwise while the tube was weakly vortexed and kept $30 \mathrm{~min}$ on ice. Cells were washed and fixed with $1 \mathrm{ml}$ of $1 \%$ paraformaldehyde/PBS containing $0.05 \%$ Tween 20 overnight at $4^{\circ} \mathrm{C}$. Fixed cells were resuspended in $1 \mathrm{ml}$ of $0.15 \mathrm{M} \mathrm{NaCl}, 4.2 \mathrm{mM}$ $\mathrm{MgCl}_{2}$, and $10 \mu \mathrm{M} \mathrm{HCl}$ containing $30 \mathrm{U} \mathrm{ml}^{-1} \mathrm{DNase} \mathrm{I}$ (Sigma-Aldrich) and then incubated for $30 \mathrm{~min}$ at room temperature. After being washed twice with PBS, cells were stained overnight at $4^{\circ} \mathrm{C}$ with FITC-conjugated anti-BrdU (BD Biosciences) and analysed with FACSCanto II.

Western blot analysis. Cell samples were lysed in $1 \times$ SDS-PAGE sample buffer sonicated, boiled and used for SDS-PAGE followed by Western blotting. The following primary polyclonal antibodies were used: rabbit anti-Bcl-6 (Cell Signaling), rabbit anti-BcL-xL (S-18), goat anti-Irf4 (M-17) and rabbit anti-Syk (N-19) (Santa Cruz Biotechnology), and rabbit $\beta$-actin (Abcam). Goat anti-rabbit IgG-HRP and rabbit anti-goat IgG-HRP (Zymed) were used as secondary antibodies. ECL western blotting detection reagents (Amersham) were used for detection.

Immunization and adoptive transfer. Purified iGB cells $\left(2 \times 10^{7}\right)$ derived from Hy10 or B1-8ki mice of C57BL/6-Ly5.1 background were injected i.v. into $\gamma$-irradiated $(6.5 \mathrm{~Gy}) \mathrm{C} 57 \mathrm{BL} / 6$ mice $\left(\mathrm{Ly} 5.2^{+}\right)$. After $\geq 30$ days, B cells purified from pooled spleens of the recipient mice were analysed by flow cytometry, as described above, and HEL- or NP-specific iMB cells, defined as CD $19^{+}$Ly5.1 ${ }^{+}$, were enumerated. To examine the immune response of these iMB cells, whole spleen cells from the mice previously immunized with OVA/complete Freund's adjuvant (CFA) or CD4 ${ }^{+} \mathrm{T}$ cells from those immunized with CGG/alum, both including memory T cells, were mixed with splenic B cells containing equal number of iMB cells and transferred into C57BL/6 mice. Twelve hours later, recipients were immunized i.v. with OVA-HEL $(20 \mu \mathrm{g})$ or $\mathrm{NP}_{40}$-CGG $(25 \mu \mathrm{g})$ in PBS and bled from the tail vein for ELISA on the indicated days after immunization.

ELISA and ELISPOT assays. NP-specific AFCs in BM or purified iGB cells were detected using an ELISPOT assay on a MultiScreen 96-well filtration plate (Millipore) coated with $10 \mu \mathrm{g} \mathrm{ml}^{-1} \mathrm{NP}_{9}$-BSA. Serially diluted cells were added to individual wells in triplicate and then incubated for $6 \mathrm{~h}$ in a humidified atmosphere at $37^{\circ} \mathrm{C}$ with $5 \% \mathrm{CO}_{2}$. Anti-NP IgG1 spots were revealed by HRP-conjugated goat anti-mouse IgG1 Ab (Southern Biotechnology) in conjunction with AEC substrate (DAKO). ELISA was performed as follows: flat-bottom 96-well plates (NUNC) were coated with $\mathrm{NP}_{9}$-BSA $\left(10 \mu \mathrm{g} \mathrm{ml}^{-1}\right)$ or HEL $\left(2 \mu \mathrm{g} \mathrm{ml}^{-1}\right.$; Sigma) and blocked with $10 \%$ FCS or $3 \%$ BSA in PBS, respectively, and serially diluted serum samples were then added to individual wells. Bound Abs were revealed by HRP-conjugated goat anti-mouse IgG1 Ab (Southern Biotechnology) in conjunction with TMB substrate (Sigma). Absorbance at $450 \mathrm{~nm}$ was measured using a Benchmark microplate reader (Bio-Rad). mAbs binding with high affinity to HEL $(\mathrm{C} 2)^{58}$ or NP $(\mathrm{C} 6)^{59}$, respectively, were used as standards.

Immunofluorescence microscopy. Immunized spleens were embedded in O.C.T. compound (Sakura), frozen in liquid nitrogen, and stored at $-80^{\circ} \mathrm{C}$. Frozen sections ( $7 \mu \mathrm{m}$ thick) were fixed in $-20^{\circ} \mathrm{C}$ acetone for $15 \mathrm{~s}$. After being washed with a buffer $(0.01 \%$ Tween 20 in PBS), the sections were pre-incubated with a staining buffer $\left(0.01 \%\right.$ Tween 20 and $3 \%$ BSA in PBS) containing $2.4 \mathrm{G} 2\left(1 \mu \mathrm{g} \mathrm{ml}^{-1}\right)$ for $30 \mathrm{~min}$ at room temperature. The sections were stained with $0.7 \mu \mathrm{g} \mathrm{ml}^{-1}$ biotinylated HEL (HEL-biotin), $5 \mu \mathrm{g} \mathrm{ml}^{-1}$ anti-B220-APC (eBioscience), Alexa-647conjugated anti-MAdCAM1, anti-Thy1.2-FITC, anti-B220-FITC (BD Biosciences) and/or goat anti-IgG1-FITC (Southern Biotechnology) as indicated followed by PE-conjugated avidin $\left(1 \mu \mathrm{g} \mathrm{ml}^{-1}\right.$; eBioscience $)$. After washing, the slides were mounted with Fluorescent Mounting medium (Dako). The samples were examined by immunofluorescence microscopy (BZ-9000; Keyence).

Quantitative RT-PCR. RNA from sorted B cells was prepared with RNeasy (Qiagen). The ReverTra Ace first-strand cDNA synthesis kit (Toyobo) was used to prepare cDNA. Quantitative real-time PCR was performed with the Power SYBR Green PCR Master Mix (Applied Biosystems) and a 7500 Fast Real-Time PCR System (Applied Biosystems) according to standard PCR conditions. Gene expression was normalized to that of Gapdh (encoding glyceraldehyde-3-phosphate dehydrogenase) and data are presented as a fold difference of the normalized values relative to that of follicular B cells by the ' $2-\Delta \Delta C T$ ' (change in cycling threshold) method. The following primer sets were used: Gapdh sense: $5^{\prime}$-ggagaaacctgccaagt atga- $3^{\prime}$; Gapdh antisense: $5^{\prime}$-ccctgttgctgtagccgtatt- $3^{\prime}$; Blimp- 1 sense: $5^{\prime}$-gaacctgctttt caagtatgctg- $3^{\prime}$; Blimp-1 antisense: $5^{\prime}$-agtgtagacttcaccgatgagg- $3^{\prime}$.

Statistical analysis. Statistical analysis was performed using the Student's $t$-test.

\section{References}

1. MacLennan, I. C. Germinal centers. Annu. Rev. Immunol. 12, 117-139 (1994).

2. Tarlinton, D. B-cell memory: are subsets necessary? Nat. Rev. Immunol. 6, 785-790 (2006).

3. Good-Jacobson, K. L. \& Shlomchik, M. J. Plasticity and heterogeneity in the generation of memory B cells and long-lived plasma cells: the influence of germinal center interactions and dynamics. J. Immunol. 185, 3117-3125 (2010).

4. McHeyzer-Williams, L. J. \& McHeyzer-Williams, M. G. Antigen-specific memory B cell development. Annu. Rev. Immunol. 23, 487-513 (2005).

5. Erazo, A. et al. Unique maturation program of the IgE response in vivo. Immunity 26, 191-203 (2007)

6. Renshaw, B. R. et al. Humoral immune responses in CD40 ligand-deficient mice. J. Exp. Med. 180, 1889-1900 (1994).

7. Foy, T. M. et al. gp39-CD40 interactions are essential for germinal center formation and the development of B cell memory. J. Exp. Med. 180, 157-163 (1994).

8. Kawabe, T. et al. The immune responses in CD40-deficient mice: impaired immunoglobulin class switching and germinal center formation. Immunity $\mathbf{1}$, 167-178 (1994).

9. Zotos, D. et al. IL-21 regulates germinal center B cell differentiation and proliferation through a B cell-intrinsic mechanism. J. Exp. Med. 207, 365-378 (2010).

10. Linterman, M. A. et al. IL-21 acts directly on B cells to regulate Bcl-6 expression and germinal center responses. J. Exp. Med. 207, 353-363 (2010).

11. Bessa, J., Kopf, M. \& Bachmann, M. F. Cutting edge: IL-21 and TLR signaling regulate germinal center responses in a B cell-intrinsic manner. J. Immunol. 184, 4615-4619 (2010).

12. Ozaki, K. et al. A critical role for IL-21 in regulating immunoglobulin production. Science 298, 1630-1634 (2002).

13. Pene, J. et al. Cutting edge: IL-21 is a switch factor for the production of IgG1 and IgG3 by human B cells. J. Immunol. 172, 5154-5157 (2004). 
14. Ozaki, K. et al. Regulation of B cell differentiation and plasma cell generation by IL-21, a novel inducer of Blimp-1 and Bcl-6. J. Immunol. 173, 5361-5371 (2004).

15. Kwon, H. et al. Analysis of interleukin-21-induced Prdm1 gene regulation reveals functional cooperation of STAT3 and IRF4 transcription factors. Immunity 31, 941-952 (2009).

16. Ettinger, R. et al. IL-21 induces differentiation of human naive and memory B cells into antibody-secreting plasma cells. J. Immunol. 175, 7867-7879 (2005).

17. Kuchen, S. et al. Essential role of IL-21 in B cell activation, expansion, and plasma cell generation during CD4+ T cell-B cell collaboration. J. Immunol. 179, 5886-5896 (2007)

18. Bryant, V. L. et al. Cytokine-mediated regulation of human B cell differentiation into Ig-secreting cells: predominant role of IL- 21 produced by CXCR5+ T follicular helper cells. J. Immunol. 179, 8180-8190 (2007).

19. Nurieva, R. I. et al. Generation of T follicular helper cells is mediated by interleukin- 21 but independent of T helper 1, 2, or 17 cell lineages. Immunity 29, 138-149 (2008).

20. Vogelzang, A. et al. A fundamental role for interleukin-21 in the generation of T follicular helper cells. Immunity 29, 127-137 (2008).

21. Yu, D. et al. The transcriptional repressor Bcl-6 directs $\mathrm{T}$ follicular helper cell lineage commitment. Immunity 31, 457-468 (2009).

22. Dent, A. L., Shaffer, A. L., Yu, X., Allman, D. \& Staudt, L. M. Control of inflammation, cytokine expression, and germinal center formation by BCL-6. Science 276, 589-592 (1997).

23. Shapiro-Shelef, M. et al. Blimp-1 is required for the formation of immunoglobulin secreting plasma cells and pre-plasma memory B cells. Immunity 19, 607-620 (2003).

24. Klein, U. et al. Transcription factor IRF4 controls plasma cell differentiation and class-switch recombination. Nat. Immunol. 7, 773-782 (2006).

25. Anderson, S. M., Tomayko, M. M., Ahuja, A., Haberman, A. M. \& Shlomchik, M. J. New markers for murine memory B cells that define mutated and unmutated subsets. J. Exp. Med. 204, 2103-2114 (2007).

26. Ridderstad, A. \& Tarlinton, D. M. Kinetics of establishing the memory B cell population as revealed by CD38 expression. J. Immunol. 160, 4688-4695 (1998).

27. Toyama, H. et al. Memory B cells without somatic hypermutation are generated from Bcl6-deficient B cells. Immunity 17, 329-339 (2002).

28. Dogan, I. et al. Multiple layers of B cell memory with different effector functions. Nat. Immunol. 10, 1292-1299 (2009).

29. Pape, K. A., Taylor, J. J., Maul, R. W., Gearhart, P. J. \& Jenkins, M. K. Different B cell populations mediate early and late memory during an endogenous immune response. Science 331, 1203-1207 (2011).

30. Black, S. J., van der Loo, W., Loken, M. R. \& Herzenberg, L. A. Expression of IgD by murine lymphocytes. Loss of surface IgD indicates maturation of memory B cells. J. Exp. Med. 147, 984-996 (1978).

31. Fehr, T. et al. Antiviral protection and germinal center formation, but impaired B cell memory in the absence of CD19. J. Exp. Med. 188, 145-155 (1998).

32. Takahashi, Y. et al. Novel role of the Ras cascade in memory B cell response. Immunity 23, 127-138 (2005).

33. Arpin, C. et al. Generation of memory B cells and plasma cells in vitro. Science 268, 720-722 (1995)

34. Choe, J., Kim, H.S., Zhang, X., Armitage, R. J. \& Choi, Y. S. Cellular and molecular factors that regulate the differentiation and apoptosis of germinal center B cells. Anti-Ig down-regulates Fas expression of CD40 ligandstimulated germinal center B cells and inhibits Fas-mediated apoptosis. J. Immunol. 157, 1006-1016 (1996).

35. Choe, J., Kim, H. S., Armitage, R. J. \& Choi, Y. S. The functional role of B cell antigen receptor stimulation and IL-4 in the generation of human memory B cells from germinal center B cells. J. Immunol. 159, 3757-3766 (1997).

36. Allen, C. D. et al. Germinal center dark and light zone organization is mediated by CXCR4 and CXCR5. Nat. Immunol. 5, 943-952 (2004).

37. Reif, K. et al. Balanced responsiveness to chemoattractants from adjacent zones determines B-cell position. Nature 416, 94-99 (2002).

38. Vikstrom, I. et al. Mcl-1 is essential for germinal center formation and B cell memory. Science 330, 1095-1099 (2010).

39. Schittek, B. \& Rajewsky, K. Maintenance of B-cell memory by long-lived cells generated from proliferating precursors. Nature 346, 749-751 (1990).

40. Allen, C. D., Okada, T., Tang, H. L. \& Cyster, J. G. Imaging of germinal center selection events during affinity maturation. Science $315,528-531$ (2007).

41. Phan, T. G., Green, J. A., Gray, E. E., Xu, Y. \& Cyster, J. G. Immune complex relay by subcapsular sinus macrophages and noncognate B cells drives antibody affinity maturation. Nat. Immunol. 10, 786-793 (2009).

42. Lam, K. P., Kuhn, R. \& Rajewsky, K. In vivo ablation of surface immunoglobulin on mature B cells by inducible gene targeting results in rapid cell death. Cell $\mathbf{9 0}$, 1073-1083 (1997).
43. Benson, M. J. et al. Distinction of the memory B cell response to cognate antigen versus bystander inflammatory signals. J. Exp. Med. 206, 2013-2025 (2009).

44. Jin, H., Carrio, R., Yu, A. \& Malek, T. R. Distinct activation signals determine whether IL-21 induces B cell costimulation, growth arrest, or Bim-dependent apoptosis. J. Immunol. 173, 657-665 (2004).

45. Kasaian, M. T. et al. IL-21 limits NK cell responses and promotes antigenspecific T cell activation: a mediator of the transition from innate to adaptive immunity. Immunity 16, 559-569 (2002).

46. Rajewsky, K., Forster, I. \& Cumano, A. Evolutionary and somatic selection of the antibody repertoire in the mouse. Science 238, 1088-1094 (1987).

47. Casali, P., Pal, Z., Xu, Z. \& Zan, H. DNA repair in antibody somatic hypermutation. Trends Immunol. 27, 313-321 (2006).

48. Good, K. L., Bryant, V. L. \& Tangye, S. G. Kinetics of human B cell behavior and amplification of proliferative responses following stimulation with IL-21. J. Immunol. 177, 5236-5247 (2006).

49. O’Connor, B. P., Cascalho, M. \& Noelle, R. J. Short-lived and long-lived bone marrow plasma cells are derived from a novel precursor population. J. Exp. Med. 195, 737-745 (2002).

50. Kwakkenbos, M. J. et al. Generation of stable monoclonal antibody-producing B cell receptor-positive human memory B cells by genetic programming. Nat. Med. 16, 123-128 (2010).

51. Allman, D. et al. BCL-6 expression during B-cell activation. Blood 87, 5257-5268 (1996).

52. Saito, M. et al. A signaling pathway mediating downregulation of BCL6 in germinal center B cells is blocked by BCL6 gene alterations in B cell lymphoma. Cancer Cell 12, 280-292 (2007).

53. Shaffer, A. L. et al. Blimp-1 orchestrates plasma cell differentiation by extinguishing the mature B cell gene expression program. Immunity 17, 51-62 (2002)

54. Ridderstad, A. \& Tarlinton, D. M. B cell memory in xid mice is long-lived despite reduced memory B cell frequency. Scand. J. Immunol. 45, 655-659 (1997).

55. Yamamoto, M. et al. BASH-deficient mice: limited primary repertoire and antibody formation, but sufficient affinity maturation and memory B cell generation, in anti-NP response. Int. Immunol. 16, 1161-1171 (2004).

56. Inamine, A. et al. Two waves of memory B-cell generation in the primary immune response. Int. Immunol. 17, 581-589 (2005).

57. Morimura, T. et al. Cell cycle arrest and apoptosis induced by Notch1 in B cells. J. Biol. Chem. 275, 36523-36531 (2000).

58. Oda, M. et al. Evaluation of the conformational equilibrium of reduced hen egg lysozyme by antibodies to the native form. Arch. Biochem. Biophys. 494, 145-150 (2010).

59. Terauchi, A. et al. A pivotal role for DNase I-sensitive regions $3 \mathrm{~b}$ and/or 4 in the induction of somatic hypermutation of IgH genes. J. Immunol. 167, 811-820 (2001).

\section{Acknowledgements}

We thank J.G. Cyster (HHMI, UCSF) and T. Okada (RCAI, RIKEN) for Hy10 mice; K. Rajewsky (Harvard University) and M. Maruyama (National Institute for Longevity Sciences) for B1-8 ki mice; T. Takemori (RCAI, RIKEN), Y. Takahashi (National Institute of Infectious Diseases), T. Tokuhisa (Chiba University) for discussion; Y. Seki (Loyola University Chicago), H. Kishimoto, A. Murakami, M. Nishimura, H. Iida, T. Hirayama (RIBS) for technical advice; Y. Kawai and A. Oda (RIBS) for mouse husbandry, and P.D. Burrows for critical comments on the manuscript. Supported in part by Grants-in-Aid for Scientific Research (B) and on priority areas (to D.K.), and for Young Scientists (to T.N.) from Ministry of Education, Culture, Sports, Science and Technology of Japan.

\section{Author contributions}

T.N. designed and supervised the experiments. T.N., K.H., T.M., M.M., S.M. and I.S. performed the experiments and analysed the data. T.A. provided essential reagents and expertise. T.N. and D.K. wrote the manuscript. D.K. designed and supervised the study. K.H. and T.M. contributed equally and deeply to the study.

\section{Additional information}

Supplementary Information accompanies this paper at http://www.nature.com/ naturecommunications

Competing financial interests: The authors declare no competing financial interests.

Reprints and permission information is available online at http://npg.nature.com/ reprintsandpermissions/

How to cite this article: Nojima, T. et al. In-vitro derived germinal centre B cell differentially generate memory B or plasma cells in vivo. Nat. Commun. 2:465 doi: 10.1038/ncomms1475 (2011) 